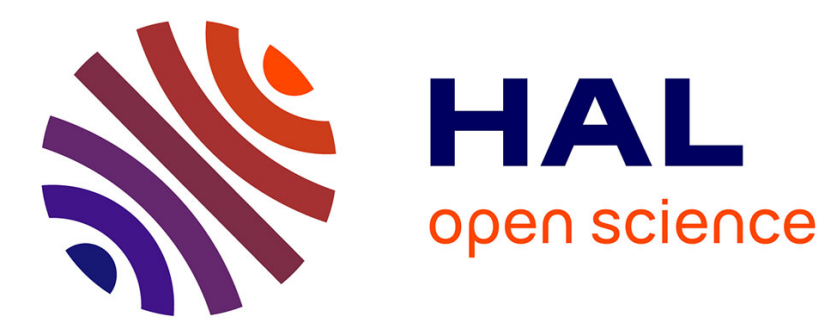

\title{
Microscopic and macroscopic instabilities in finitely strained porous elastomers
}

J. C. Michel, O. Lopez-Pamies, P. Ponte Castañeda, N. Triantafillydis

\section{To cite this version:}

J. C. Michel, O. Lopez-Pamies, P. Ponte Castañeda, N. Triantafillydis. Microscopic and macroscopic instabilities in finitely strained porous elastomers. Journal of the Mechanics and Physics of Solids, 2006, 55 (5), pp.900-938. 10.1016/j.jmps.2006.11.006 . hal-00111501

\section{HAL Id: hal-00111501 https://hal.science/hal-00111501}

Submitted on 1 Mar 2019

HAL is a multi-disciplinary open access archive for the deposit and dissemination of scientific research documents, whether they are published or not. The documents may come from teaching and research institutions in France or abroad, or from public or private research centers.
L'archive ouverte pluridisciplinaire HAL, est destinée au dépôt et à la diffusion de documents scientifiques de niveau recherche, publiés ou non, émanant des établissements d'enseignement et de recherche français ou étrangers, des laboratoires publics ou privés. 


\title{
Microscopic and macroscopic instabilities in finitely strained porous elastomers
}

\author{
J.C. Michel ${ }^{\mathrm{a}}$, O. Lopez-Pamies ${ }^{\mathrm{b}, \mathrm{c}}$, \\ P. Ponte Castañeda ${ }^{\mathrm{b}, \mathrm{c}}$, N. Triantafyllidis ${ }^{\mathrm{d}, *}$ \\ ${ }^{a}$ Laboratory of Mechanics and Acoustics, C.N.R.S. UPR7051, 31 Chemin Joseph Aiguier, Marseille 13402, France \\ ${ }^{\mathrm{b}}$ Laboratory of Solid Mechanics, C.N.R.S. UMR7649, Ecole Polytechnique, Palaiseau 91128, France \\ ${ }^{\mathrm{c}}$ Department of Mechanical Engineering and Applied Mechanics, The University of Pennsylvania, Philadelphia, \\ PA 19104-6315, USA \\ ${ }^{\mathrm{d}}$ Department of Aerospace Engineering, The University of Michigan, Ann Arbor, Michigan 48109-2140, USA
}

The present work is an in-depth study of the connections between microstructural instabilities and their macroscopic manifestations - as captured through the effective properties - in finitely strained porous elastomers. The powerful second-order homogenization ( $\mathrm{SOH}$ ) technique initially developed for random media, is used for the first time here to study the onset of failure in periodic porous elastomers and the results are compared to more accurate finite element method (FEM) calculations. The influence of different microgeometries (random and periodic), initial porosity, matrix constitutive law and macroscopic load orientation on the microscopic buckling (for periodic microgeometries) and macroscopic loss of ellipticity (for all microgeometries) is investigated in detail. In addition to the above-described stability-based onset-of-failure mechanisms, constraints on the principal solution are also addressed, thus giving a complete picture of the different possible failure mechanisms present in finitely strained porous elastomers.

Keywords: Porous material; Elastic material; Finite strain; Stability and bifurcation; Finite elements

\footnotetext{
*Corresponding author.

E-mail address: nick@umich.edu (N. Triantafyllidis).
} 


\section{Introduction and motivation}

Failure in composite materials is a fundamental as well as an extremely diverse issue in solid mechanics. Questions about what constitutes failure, when does failure start and whether it is possible to predict the onset of failure by investigating the effective (homogenized) properties of the solid, are problems of fundamental interest for all composites. The infinite variety of matrix materials and microstructures, which result in a multitude of possible failure mechanisms, is the reason for the problem's extraordinary diversity.

In the interest of (relative) simplicity, and motivated by considerable recent advances in calculating the effective properties of finitely strained non-linear solids, attention is focused here on porous elastomers. This particular class of composites is of considerable technological interest and enjoys a wide range of applications as foams (random microstructure) or honeycombs (periodic microstructure). A brief review of the relevant literature in finitely strained porous elastomers is presented here, with particular emphasis on the associated stability issues for the case of random as well as periodic microgeometries.

An important early contribution to the field, which is credited with starting the stability discussions in porous elastomers, is the experimental work of Blatz and Ko (1962). These researchers performed experiments on polyurethane rubber with a random distribution of voids of about $40 \mu \mathrm{m}$ in diameter and an approximate volume fraction of about $50 \%$. The failure mechanism associated with this elastomer was first pointed by Knowles and Sternberg (1975) who showed that the incremental equilibrium equations of this material lose ellipticity at adequately large strain levels. These works were within the framework of continuum mechanics and did not address the connections between the existence of a microstructure and the macroscopic loss of rank-one convexity of the continuum energy density.

Independently, considerable effort has been devoted to predict the effective properties of hyperelastic composites with random microstructures, starting with the work of Hill (1972). The homogenization of these solids presents serious technical challenges which were addressed in a series of papers by P. Ponte Castañeda and co-workers, starting with Ponte Castañeda (1989). From the increasingly accurate homogenization schemes which have been subsequently devised, of particular interest here is the second-order homogenization (SOH) method (Ponte Castañeda, 1996, 2002). This method has been applied recently to particle-reinforced elastomers by Lopez-Pamies and Ponte Castaneda (2003, 2004a), as well as to porous elastomers by Lopez-Pamies and Ponte Castañeda (2004b). These models are sophisticated enough as to allow for modeling of the evolving microstructure and are thus successful in predicting the loss of rank-one convexity in homogenized porous elastomers.

Motivated by the work of Knowles and Sternberg (1975) and in parallel with the homogenization studies for finite strain elastomers, the connection between porosity at the microscopic level and loss of ellipticity at the macroscopic scale became the object of considerable attention. The work of Abeyaratne and Triantafyllidis (1984) first showed how by homogenizing the tangent moduli of a finitely strained periodic porous elastomer with a strictly rank-one convex matrix, one can obtain at finite strain a macroscopically non-elliptic material with the same tendencies exhibited by the Blatz-Ko solid. Moreover, an intimate connection was discovered between the onset of microscopic buckling and the 
corresponding loss of ellipticity of the incremental moduli in the homogenized solid. This was first shown by Triantafyllidis and Maker (1985) for an incompressible, hyperelastic layered composite in plane strain. Subsequent work by Geymonat et al. (1993) established a rigorous connection between bifurcation instability at the microscopic level and loss of rank-one convexity of the homogenized moduli in finitely strained periodic elastomers of infinite extent. More specifically, it was shown that if the wavelength of the bifurcation eigenmode is infinite (compared to the unit cell size), the corresponding instability of the periodic principal solution can be detected as a loss of ellipticity of the corresponding onecell homogenized tangent moduli of the solid.

Based on these general results, Triantafyllidis and Bardenhagen (1996) defined the onsetof-failure surfaces in stress and strain space for periodic solids of infinite extent, a concept which was subsequently applied among other solids to the case of trusses by Schraad and Triantafyllidis (1997), biaxially compressed aluminum honeycomb by Triantafyllidis and Schraad (1998), fiber-reinforced composites under combined normal and shear strains by Nestorović and Triantafyllidis (2004), to three-dimensional Kelvin foams (Gong et al., 2005) and more recently to periodic porous and particulate elastomers by Triantafyllidis et al. (2006).

The present paper, a logical continuation and merger of the above-described parallel efforts, is an in-depth study of the connections between microstructure and macroscopic failure in random as well as in periodic porous elastomers. More specifically, the powerful $\mathrm{SOH}$ technique previously applied to study random media, is hereby applied for the first time to study the onset of failure in periodic porous elastomers and the results are compared to more accurate finite element calculations. The influence of different microgeometries (random and periodic), initial porosity and matrix constitutive law on the microscopic buckling (for periodic microstructures) and macroscopic loss of ellipticity (for arbitrary microstructures) is investigated in detail. In addition to the above-described stability-based onset-of-failure mechanisms, a number of additional constraints in the effective response of porous media are also addressed, thus giving a complete picture of the different possible failure mechanisms present in finitely strained porous elastomers.

The presentation of the work is organized as follows: Section 2 deals with the general, three-dimensional theoretical considerations for the effective properties of porous elastomers, the relation between microscopic instabilities and macroscopic loss of ellipticity (for periodic microstructures) as well as the definition of the onset-of-failure surfaces. The description of the loading path in plane strain and the presentation of the different (two-dimensional) calculation methods (the SOH method with associated linear comparison solid, as well as the finite element method (FEM)) are given in Section 3. The specific matrix constitutive choices and the results of the calculations are presented and discussed in Section 4 followed by the conclusion in Section 5. Finally, the additional constraints on the effective response of porous elastomers (void surface instability, percolation, strain lock-up and pore closure/self-contact) are addressed in Appendix A while the expressions for the microstructural tensor $\mathbf{P}$, which is required for the homogenization calculations, are given for different microgeometries in Appendix B.

\section{Theoretical considerations}

As stated in the Introduction, the goal of this work is to study the instabilities of porous, elastic solids subjected to finite-strain loading conditions. Of particular interest is the 
microscopic (local) instability information that can be extracted by investigating the macroscopic (homogenized) properties of porous elastomers. Also of interest is the influence of microgeometry on the stability of these solids. The concepts introduced in this Section are general and apply to two- or three-dimensional porous elastomers with pointwise rank-one convex matrix constitutive laws.

The first subsection deals with the macroscopic properties of porous elastomers and the related concept of macroscopic stability, which is based on the fact that the equilibrium solution of a representative volume element (RVE) under Dirichlet boundary conditions is a local minimizer of the RVE's energy. This criterion leads to the strong ellipticity of the homogenized solid's incremental moduli or, equivalently, to the strict rank-one convexity of the homogenized stored-energy function.

The second subsection pertains to the stability of periodic porous elastomers. For solids with periodic microstructures, a simple unit cell can be easily identified and more accurate numerical homogenization calculations can be accomplished based on this unit cell. The question that naturally arises is whether for a given macroscopic load, the energy minimizing solution is periodic with period the chosen unit cell, or whether a different energy minimizer exists which is periodic on a larger cell. To this end, the concept of microscopic stability is introduced in the second subsection, which is based on the examination of all bounded perturbations in the infinite solid about the periodic equilibrium solution under consideration. The microscopic stability investigation makes use of the chosen small unit cell in conjunction with a Bloch-wave technique.

Following the above stability definitions, the concept of a microscopic and a macroscopic onset-of-failure surface in macroscopic load space is introduced in the third subsection. For random microgeometries only the macroscopic onset-of-instability surface can be defined. For periodic microstructures, both onset-of-failure surfaces (i.e., the microscopic and the macroscopic surfaces) can be found and the former surface lies by definition inside the latter. The microscopic onset-of-failure surfaces can also be used to characterize the domain in macroscopic load space where the homogenized properties based on calculations using the chosen unit cell are valid and coincide with the homogenized properties calculated using an infinite number of cells.

\subsection{Macroscopic properties and stability of porous elastomers}

Consider a porous elastomer made up of voids that are distributed, either with periodic or random microstructure, in an elastomeric matrix phase. A specimen of this porous material is assumed to occupy a volume $\Omega_{0}$ in the reference configuration, in such a way that the typical size of the voids is much smaller than the size of the specimen and the scale of variation of the applied loading. Thus, in the homogenization limit (i.e., in the limit as the size of the pores goes to zero), $\Omega_{0}$ can be identified with a RVE of the porous elastomer.

Some useful notation is in order at this point; the average of a field quantity $\mathbf{f}$, denoted by $\langle\mathbf{f}\rangle$ is defined by

$$
\langle\mathbf{f}\rangle \equiv \frac{1}{\Omega_{0}} \int_{\Omega_{0}} \mathbf{f}(\mathbf{X}) \mathrm{d} \mathbf{X} .
$$

Material points in the solid are identified by their initial position vector $\mathbf{X}$, while the current position vector of the same point is denoted by $\mathbf{x}$. The displacement of each material point $\mathbf{X}$ is denoted by $\mathbf{u}$, such that $\mathbf{u} \equiv \mathbf{X}-\mathbf{X}$. The deformation gradient $\mathbf{F}$ at $\mathbf{X}$, a 
quantity that measures the deformation in the neighborhood of $\mathbf{X}$, is defined as

$$
\mathbf{F} \equiv \mathbf{x} \nabla=\mathbf{I}+\mathbf{u} \nabla \quad\left(F_{i j} \equiv \partial x_{i} / \partial X_{j}=\delta_{i j}+\partial u_{i} / \partial X_{j}\right) .
$$

The constitutive behavior of the hyperelastic matrix phase, which occupies the subdomain $\Omega_{0}^{(1)}$, is characterized by a stored-energy function $W^{(1)}$ that is a non-convex function of the deformation gradient $\mathbf{F}$. The constitutive behavior of the vacuous phase, which occupies the subdomain $\Omega_{0}^{(2)}$, is described by the stored-energy function $W^{(2)}=0$. Thus, the local energy function of this two-phase system may be written as

$$
W(\mathbf{X} ; \mathbf{F})=\sum_{r=1}^{2} \chi_{0}^{(r)}(\mathbf{X}) W^{(r)}(\mathbf{F}) .
$$

Here the functions $\chi_{0}^{(1)}$, equal to 1 if the position vector $\mathbf{X} \in \Omega_{0}^{(1)}$ and zero otherwise, and $\chi_{0}^{(2)}$, equal to 1 if the position vector $\mathbf{X} \in \Omega_{0}^{(2)}$ and zero otherwise, describe the distribution of the two phases in the reference configuration of the hyperelastic porous solid. Since there is really only one phase in this system, and $\chi_{0}^{(2)}=1-\chi_{0}^{(1)}$, one can alternatively write

$$
W(\mathbf{X} ; \mathbf{F})=\chi_{0}(\mathbf{X}) W(\mathbf{F}),
$$

where $\chi_{0}$ and $W$ are used instead of $\chi_{0}^{(1)}$ and $W^{(1)}$, for simplicity.

The characteristic function $\chi_{0}$ may be periodic or random. In the first case, the dependence of $\chi_{0}$ on the position vector $\mathbf{X}$ is completely determined once a unit cell $D$ has been specified. In the second case, the dependence of $\chi_{0}$ on $\mathbf{X}$ is not known precisely, and the microstructure is only partially defined in terms of the $n$-point statistics of the system. Here, use will be made of information up to only two-point statistics in order to be able to take advantage of linear homogenization estimates that are available from the literature. It is noted that the initial volume fraction of the matrix phase is given by $\left\langle\chi_{0}\right\rangle$, in such a way that the initial value of the porosity will be determined by $f_{0}=1-\left\langle\chi_{0}\right\rangle$.

The stored-energy function of the matrix phase will, of course, be assumed to be objective in the sense that $W\left(Q_{i k} F_{k j}\right)=W\left(F_{i j}\right)$ for all proper orthogonal $\mathbf{Q}$ and arbitrary deformation gradients $\mathbf{F}$. Making use of the polar decomposition $F_{i j}=R_{i k} U_{k j}$, where $\mathbf{U}$ is the right stretch tensor and $\mathbf{R}$ is the rotation tensor, it follows, in particular, that $W(\mathbf{F})=W(\mathbf{U})$. The constitutive relation of the matrix material is

$$
\mathbf{S}=\frac{\partial W}{\partial \mathbf{F}}(\mathbf{F}) \quad\left(S_{i j}=\frac{\partial W}{\partial F_{i j}}(\mathbf{F})\right),
$$

where $\mathbf{S}$ denotes the nominal stress tensor (the transpose of the first Piola-Kirchhoff stress tensor). ${ }^{1}$ Note that sufficient smoothness is assumed for $W$ on $\mathbf{F}$. It is also useful to define the local elasticity, or tangent modulus tensor of the matrix material via

$$
\mathbf{L}(\mathbf{F}) \equiv \frac{\partial^{2} W}{\partial \mathbf{F} \partial \mathbf{F}}(\mathbf{F}) \quad\left(L_{i j k l}(\mathbf{F}) \equiv \frac{\partial^{2} W}{\partial F_{i j} \partial F_{k l}}(\mathbf{F})\right) .
$$

${ }^{1}$ The definitions of stress measures adopted follow Malvern (1969). 
It is further assumed that the matrix material is strongly elliptic, or strictly rank-one convex, namely ${ }^{2}$ :

$$
B_{\mathbf{L}}(\mathbf{F}) \equiv \min _{\|\mathbf{a}\|=\|\mathbf{n}\|=1}\left\{a_{i} n_{j} L_{i j k l}(\mathbf{F}) a_{k} n_{l}\right\}>0 .
$$

The physical meaning of the above requirement is that the material never admits solutions with discontinuous deformation gradients within the given phase.

Following Hill (1972), under the above-mentioned separation of length scales hypothesis, the effective stored-energy function $\widetilde{W}$ of the porous elastomer is defined by ${ }^{3}$

$$
\widetilde{W}(\overline{\mathbf{F}}) \equiv \min _{\mathbf{F} \in \mathscr{K}(\overline{\mathbf{F}})}\langle W(\mathbf{X} ; \mathbf{F})\rangle
$$

where $\mathscr{K}$ denotes the set of admissible deformation gradients:

$$
\mathscr{K}(\overline{\mathbf{F}})=\left\{\mathbf{F} \mid F_{i j}=\partial x_{i}(\mathbf{X}) / \partial X_{j} \text { for } \mathbf{X} \in \Omega_{0}, x_{i}=\bar{F}_{i j} X_{j} \text { for } \mathbf{X} \in \partial \Omega_{0}\right\} .
$$

Note that $\widetilde{W}$ represents the minimum elastic energy stored in the entire RVE when subjected to an affine displacement boundary condition that is consistent with the average deformation condition $\langle\mathbf{F}\rangle=\overline{\mathbf{F}}$. Moreover, from definition (2.8) and the objectivity of $W$, it can be shown that $\widetilde{W}$ is objective but in general $\widetilde{W} \neq \bar{W}$. It is also noted for later reference that $\widetilde{W}$ is quasi-convex, and therefore, rank-one convex, but not necessarily strictly so, as discussed later.

Consequently, the global or macroscopic constitutive relation for the porous solid can be shown to be

$$
\overline{\mathbf{S}}=\frac{\partial \widetilde{W}}{\partial \overline{\mathbf{F}}} \equiv \widetilde{\mathbf{S}},
$$

where $\overline{\mathbf{S}}=\langle\mathbf{S}\rangle$ is the average stress in the porous elastomer and $\widetilde{\mathbf{S}}$ is defined to be the effective stress. From here on, both $\langle\mathbf{f}\rangle$ and $\overline{\mathbf{f}}$ notations for the average of a field quantity $\mathbf{f}$ are employed interchangeably according to convenience. In analogy with the local elasticity tensor defined in (2.6), the macroscopic (or effective) elasticity tensor is defined:

$$
\widetilde{\mathbf{L}}(\overline{\mathbf{F}}) \equiv \frac{\partial^{2} \widetilde{W}}{\partial \overline{\mathbf{F}} \partial \overline{\mathbf{F}}}(\overline{\mathbf{F}}),
$$

where unlike the stress case in (2.9), $\widetilde{\mathbf{L}} \neq \overline{\mathbf{L}}$.

The macroscopic stability of the solid at $\overline{\mathbf{F}}$ is measured by the effective coercivity constant $\widetilde{B}(\overline{\mathbf{F}})$, which is defined, in terms of $\widetilde{\mathbf{L}}(\overline{\mathbf{F}})$, in an analogous way to its local counterpart (2.7), by

$$
\widetilde{B}(\overline{\mathbf{F}}) \equiv \min _{\|\mathbf{a}\|=\|\mathbf{n}\|=1}\left\{a_{i} n_{j} \widetilde{L}_{i j k l}(\overline{\mathbf{F}}) a_{k} n_{l}\right\}
$$

According to (2.11), the porous solid is defined to be macroscopically stable if $\widetilde{B}(\overline{\mathbf{F}})>0$, i.e., if it is strictly rank-one convex. One of the issues of interest in this work is under what conditions it can lose strict rank-one convexity.

\footnotetext{
${ }^{2}$ Einstein's summation convention is adopted with repeated Latin indices summed from 1 to 3 in this section which deals with three-dimensional solids.

${ }^{3}$ Here and subsequently the symbol $(\widetilde{\mathbf{f}})$ implies effective properties associated with the field quantity f, as opposed to the symbol ( $(\overline{\mathbf{f}})$ which implies average properties associated with the same field.
} 


\subsection{Microscopic instabilities in periodic porous elastomers}

Consider a porous elastomer whose periodic, stress-free state is used as the undeformed reference configuration. Without loss of generality, the porous solid can be thought as resulting by periodic repetition along each coordinate direction of a fundamental building block $D$ (with boundary $\partial D$ ), which is termed the unit cell. Without loss of generality $D$ is assumed to be a parallelepiped of dimension $L_{i}$ along the direction $X_{i}$. Then, the distribution of the material is characterized by a $D$-periodic characteristic function:

$$
\chi_{0}\left(X_{1}, X_{2}, X_{3}\right)=\chi_{0}\left(X_{1}+k_{1} L_{1}, X_{2}+k_{2} L_{2}, X_{3}+k_{3} L_{3}\right),
$$

where $k_{1}, k_{2}, k_{3}$ are arbitrary integers, and $L_{1}, L_{2}, L_{3}$, the unit cell dimensions.

The primary objective of this work is to determine the macroscopic properties of the porous solid and the stability information they carry. For hyperelastic porous solids with periodic microstructure, it is known (Braides, 1985; Müller, 1987) that the computation of the effective stored-energy function $\widetilde{W}$, as determined by relation (2.8), cannot be simplified further, as a consequence of the lack of convexity of the local stored-energy function $W$. Recall that, for a periodic medium, the computation of the effective storedenergy function $\widetilde{W}$, as determined by relation (2.8), can be reduced to a computation on the unit cell, provided that the local stored-energy function $W$ be convex (Marcellini, 1978). Unfortunately, actual elastomers do not have convex energy functions. However, as will be discussed in further detail below, it is still useful in this context to define the one-cell effective energy function $\widehat{W}$ via the expression:

$$
\widehat{W}(\overline{\mathbf{F}}) \equiv \min _{\mathbf{u}^{\prime} \in D_{\sharp}}\left\{\frac{1}{|D|} \int_{D} W\left(\mathbf{X} ; \overline{\mathbf{F}}+\mathbf{u}^{\prime} \nabla\right) \mathrm{d} \mathbf{X}\right\},
$$

where by $D_{\#}$ is denoted the set of all $D$-periodic fluctuation functions $\mathbf{u}^{\prime}$, i.e., zero-average displacement functions that have the same values on opposite faces of the unit cell $D$. Since the macroscopic deformation gradient is given by $\overline{\mathbf{F}}$, the local fluctuation field is $u_{i}^{\prime}=u_{i}+X_{i}-\bar{F}_{i j} X_{j}$. Attention is focused only on macroscopic deformations $\overline{\mathbf{F}}$ for which such a fluctuation field, denoted by $\mathbf{u}_{\bar{F}}^{\prime}$, exists and corresponds to a stable equilibrium solution of the unit-cell deformation problem:

$$
\begin{aligned}
& \int_{D} \frac{\partial W}{\partial F_{i j}}\left(\mathbf{X} ; \overline{\mathbf{F}}+\mathbf{u}_{\bar{F}}^{\prime} \nabla\right) \delta u_{i, j} \mathrm{~d} \mathbf{X}=0, \\
& \beta_{D} \equiv \min _{\mathbf{u}^{\prime} \in D_{\#}}\left\{\int_{D} \frac{\partial^{2} W}{\partial F_{i j} \partial F_{k l}}\left(\mathbf{X} ; \overline{\mathbf{F}}+\mathbf{u}_{\bar{F}}^{\prime} \nabla\right) u_{i, j}^{\prime} u_{k, l}^{\prime} \mathrm{d} \mathbf{X} / \int_{D} u_{i, j}^{\prime} u_{i, j}^{\prime} \mathrm{d} \mathbf{X}\right\}>0,
\end{aligned}
$$

where $\delta \mathbf{u}$ is any arbitrary $D$-periodic fluctuation field. The first of the above equations indicates that $\mathbf{u}_{\bar{F}}^{\prime}$ is an extremum of the unit-cell energy $\widehat{W}(\overline{\mathbf{F}})$, and the second that it corresponds to a local minimum of this energy.

Although according to (2.7) the material is at each point strictly rank-one convex, this property does not usually hold for the homogenized porous solid (see Abeyaratne and Triantafyllidis, 1984). The search for the macroscopic deformations $\overline{\mathbf{F}}$ for which the homogenized solid characterized by $\widehat{W}(\overline{\mathbf{F}})$ loses its strict rank-one convexity is addressed next. To this end, one needs to investigate the one-cell homogenized moduli tensor $\widehat{\mathbf{L}}(\overline{\mathbf{F}})$, 
defined by

$$
\widehat{\mathbf{L}}(\overline{\mathbf{F}}) \equiv \frac{\partial^{2} \widehat{W}}{\partial \overline{\mathbf{F}} \partial \overline{\mathbf{F}}}(\overline{\mathbf{F}}), \quad \widehat{W}(\overline{\mathbf{F}})=\frac{1}{|D|} \int_{D} W\left(\mathbf{X} ; \overline{\mathbf{F}}+\mathbf{u}_{\bar{F}}^{\prime} \nabla\right) \mathrm{d} \mathbf{X} .
$$

When an explicit expression for $\widehat{W}$ exists, the homogenized moduli are calculated by taking the second derivative with respect to $\overline{\mathbf{F}}$ of $\widehat{W}$ given in (2.16). For the case of regular microgeometries where the unit-cell problem - as defined in (2.14) - is solved numerically using an FEM technique, a different calculation strategy, which is based on the interchange between the homogenization and linearization steps, is employed.

Thus, for a solid with a linearized response characterized by its tangent moduli $\mathbf{L}(\mathbf{X})$, where $\mathbf{L}$ is a $D$-periodic function of $\mathbf{X}$, the homogenized tangent modulus tensor $\mathbf{L}^{\mathrm{H}}$ is uniquely defined by (see Geymonat et al., 1993)

$$
G_{i j} L_{i j k l}^{\mathrm{H}} G_{k l} \equiv \min _{\mathbf{u}^{\prime} \in D_{\#}}\left\{\frac{1}{|D|} \int_{D} L_{i j k l}(\mathbf{X})\left(G_{i j}+u_{i, j}^{\prime}\right)\left(G_{k l}+u_{k, l}^{\prime}\right) \mathrm{d} \mathbf{X}\right\},
$$

where $\mathbf{G}$ is an arbitrary second-order tensor. A formal calculation based on (2.17) shows that the components of the homogenized tangent moduli are given by

$$
L_{p q r s}^{\mathrm{H}}=\frac{1}{|D|} \int_{D} L_{i j k l}(\mathbf{X})\left(\delta_{i p} \delta_{j q}+\stackrel{p q}{\chi}_{i, j}\right)\left(\delta_{k r} \delta_{l s}+\stackrel{r s}{\chi}_{k, l}\right) \mathrm{d} \mathbf{X}
$$

where the characteristic functions $\stackrel{p q}{\chi} \in D_{\#}$ are $D$-periodic fluctuations defined by

$$
\int_{D} L_{i j k l}(\mathbf{X})\left(\delta_{i p} \delta_{j q}+\stackrel{p q}{\chi}_{i, j}\right) \delta u_{k, l} \mathrm{~d} \mathbf{X}=0
$$

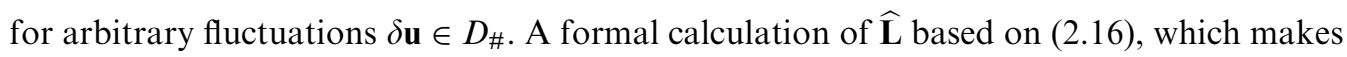
use of (2.14), shows that (see Geymonat et al., 1993) the above-defined linearization and homogenization operations commute, and therefore

$$
\widehat{\mathbf{L}}=\mathbf{L}^{\mathrm{H}} \text {. }
$$

It also follows from the same calculations that the characteristic functions ${ }^{p q} \chi$ (defined in (2.19)) involved in the determination of $\mathbf{L}^{\mathrm{H}}$ are the $\overline{\mathbf{F}}$ derivatives of the fluctuation functions $\mathbf{u}_{\bar{F}}^{\prime}$, namely:

$$
\stackrel{p q}{\chi}=\frac{\partial \mathbf{u}_{\bar{F}}^{\prime}}{\partial \bar{F}_{p q}} .
$$

By definition, the one-cell homogenized energy $\widehat{W}$ requires minimization of the energy over a single unit cell. However, it is possible that, by minimizing the energy over larger domains containing several unit cells, a lower value can be found for the energy per volume of these larger samples. The corresponding fluctuation fields are periodic over much larger (possibly infinite) domains $\mathbf{k} D$, where $\mathbf{k} D$ denotes a super-cell of dimensions $k_{i} L_{i}$ in each direction. Hence, a fully consistent definition (see Müller, 1987) of the homogenized energy $\widetilde{W}$ requires the consideration of fluctuations $\mathbf{u}^{\prime}$ that are $\mathbf{k} D$-periodic. Thus, for a periodic hyperelastic medium, the general expression (2.8) specializes to

$$
\widetilde{W}(\overline{\mathbf{F}}) \equiv \inf _{\mathbf{k} \in N^{3}}\left\{\min _{\mathbf{u}^{\prime} \in \mathbf{k} D_{\#}}\left\{\frac{1}{|\mathbf{k} D|} \int_{\mathbf{k} D} W\left(\mathbf{X} ; \overline{\mathbf{F}}+\mathbf{u}^{\prime} \nabla\right) \mathrm{d} \mathbf{X}\right\}\right\} .
$$


From the definitions in (2.13) and (2.22), one can easily conclude that $\widetilde{W}(\overline{\mathbf{F}}) \leqslant \widehat{W}(\overline{\mathbf{F}})$. The equality holds when the infimum is a minimum occurring at $\mathbf{k}=(1,1,1)$, i.e., when the onecell minimizing fluctuation displacement $\mathbf{u}_{\bar{F}}^{\prime}$ is also the minimizing fluctuation displacement for any super-cell $\mathbf{k} D$.

For small strains (near $\overline{\mathbf{F}}=\mathbf{I})$, one expects that $\widetilde{W}(\overline{\mathbf{F}})=\widehat{W}(\overline{\mathbf{F}})$, but as the macroscopic strain increases, eventually, $\widetilde{W}(\overline{\mathbf{F}})<\widehat{W}(\overline{\mathbf{F}})$. It is always possible to calculate, exactly as well as approximately, the one-cell homogenized energy $\widehat{W}(\overline{\mathbf{F}})$ and the corresponding macroscopic moduli $\widehat{\mathbf{L}}(\overline{\mathbf{F}})$. However, it is practically impossible to calculate the correct homogenized energy $\widetilde{W}(\overline{\mathbf{F}})$, in view of the infinity of the required domain of its definition $(\mathbf{k} D$ with $\|\mathbf{k}\| \rightarrow \infty)$. Therefore, it is important to establish the region of macroscopic strain space where the one-cell homogenized energy is the correct one $(\widehat{W}(\overline{\mathbf{F}})=\widetilde{W}(\overline{\mathbf{F}}))$. To this end, and in an analogous way to (2.15), one can define the coercivity constant $\beta(\overline{\mathbf{F}})$ for the infinite domain $\left(\Omega_{0}=R^{3}\right)$ :

$$
\begin{aligned}
& \beta(\overline{\mathbf{F}}) \equiv \inf _{\mathbf{k} \in N^{3}} \beta_{\mathbf{k} D}(\overline{\mathbf{F}}), \\
& \beta_{\mathbf{k} D}(\overline{\mathbf{F}}) \equiv \min _{\mathbf{u}^{\prime} \in \mathbf{k} D_{\#}}\left\{\int_{\mathbf{k} D} \frac{\partial^{2} W}{\partial F_{i j} \partial F_{k l}}\left(\mathbf{X} ; \overline{\mathbf{F}}+\mathbf{u}_{\bar{F}}^{\prime} \nabla\right) u_{i, j}^{\prime} u_{k, l}^{\prime} \mathrm{d} \mathbf{X} / \int_{\mathbf{k} D} u_{i, j}^{\prime} u_{i, j}^{\prime} \mathrm{d} \mathbf{X}\right\} .
\end{aligned}
$$

As shown by Geymonat et al. (1993), a necessary condition for $\widetilde{W}(\overline{\mathbf{F}})=\widehat{W}(\overline{\mathbf{F}})$ is that $\beta(\overline{\mathbf{F}})>0$. Fortunately, unlike the computation of $\widetilde{W}(\overline{\mathbf{F}})$, the determination of the coercivity constant $\beta(\overline{\mathbf{F}})$ requires only calculations on the unit cell $D$, as will be seen next. Thus, using the Bloch-wave representation theorem, it was proved by Geymonat et al. (1993) that the eigenmode $\mathbf{v}$ corresponding to $\beta(\overline{\mathbf{F}})$ can always be put in the form

$$
\mathbf{v}(\mathbf{X})=\mathbf{u}^{\prime}(\mathbf{X}) \exp \left(\mathrm{i} \omega_{k} X_{k}\right), \quad \mathbf{u}^{\prime} \in D_{\#} ; \quad \boldsymbol{\omega} \equiv\left(\omega_{1}, \omega_{2}, \omega_{3}\right), \quad 0 \leqslant \omega_{1} L_{1}, \omega_{2} L_{2}, \omega_{3} L_{3}<2 \pi,
$$

and hence that the coercivity constant $\beta(\overline{\mathbf{F}})$ is determined from

$$
\beta(\overline{\mathbf{F}}) \equiv \inf _{\boldsymbol{\omega}}\left\{\min _{\mathbf{u}^{\prime} \in D_{\#}}\left\{\int_{D} \frac{\partial^{2} W}{\partial F_{i j} \partial F_{k l}}\left(\mathbf{X} ; \overline{\mathbf{F}}+\mathbf{u}_{F}^{\prime} \nabla\right) v_{i, j}^{\star} v_{k, l} \mathrm{~d} \mathbf{X} / \int_{D} v_{i, j}^{\star} v_{i, j} \mathrm{~d} \mathbf{X}\right\}\right\},
$$

with $\mathbf{v}$ given by $(2.25)_{1}$. Here, $\mathbf{v}^{\star}$ is the complex conjugate of the field $\mathbf{v}$.

The Euler-Lagrange equations and free-surface boundary conditions corresponding to the above eigenvalue problem (2.26) are

$$
\left(L_{i j k l}\left(\mathbf{X} ; \overline{\mathbf{F}}+\mathbf{u}_{\bar{F}}^{\prime} \nabla\right) v_{k, l}-\beta(\overline{\mathbf{F}}) v_{i, j}\right)_{, j}=0 \quad\left(L_{i j k l}\left(\mathbf{X} ; \overline{\mathbf{F}}+\mathbf{u}_{\bar{F}}^{\prime} \nabla\right) v_{k, l}-\beta(\overline{\mathbf{F}}) v_{i, j}\right) N_{j}=0,
$$

where $\mathbf{N}$ is the outward normal to the free surface of the pores and the eigenmode $\mathbf{v}$ is given in terms of the Bloch representation theorem (2.25). Of course, the same equations are applicable for the eigenmode corresponding to $\beta_{\mathbf{k} D}$, defined in (2.24), and also for the eigenmode corresponding to $\beta_{D}$, defined in (2.15).

Of particular interest here is $\beta_{0}(\overline{\mathbf{F}})$, the long-wavelength limit $(\omega \rightarrow 0)$ of the above expression (2.26), defined as

$$
\beta_{0}(\overline{\mathbf{F}}) \equiv \liminf _{\boldsymbol{\omega} \rightarrow \mathbf{0}}\left\{\min _{\mathbf{u}^{\prime} \in D_{\sharp}}\left\{\int_{D} \frac{\partial^{2} W}{\partial F_{i j} \partial F_{k l}}\left(\mathbf{X} ; \overline{\mathbf{F}}+\mathbf{u}_{\bar{F}}^{\prime} \nabla\right) v_{i, j}^{\star} v_{k, l} \mathrm{~d} \mathbf{X} / \int_{D} v_{i, j}^{\star} v_{i, j} \mathrm{~d} \mathbf{X}\right\}\right\},
$$


which, as will be subsequently discussed, when it vanishes, signals the loss of strict rankone convexity of the one-cell homogenized stored energy $\widehat{W}(\overline{\mathbf{F}})$.

The use of lim inf $\boldsymbol{\omega}_{\boldsymbol{\omega} \rightarrow \mathbf{0}}$ in the above expression merits further explanation. As seen from (2.25) two different types of eigenmodes exist in the neighborhood of $\boldsymbol{\omega}=\mathbf{0}$; the strictly $D$ periodic ones, for which $\boldsymbol{\omega}=\mathbf{0}$, and the long-wavelength modes, for which $\boldsymbol{\omega} \rightarrow \mathbf{0}$. Depending on the case, the lowest value of the ratio of the two quadratic functionals in (2.28) can occur for long wavelength modes, in which case the limit $\boldsymbol{\omega} \rightarrow \mathbf{0}$ is a singular one depending on the ratio of the $\omega$ components, thus justifying the use of the lim inf in (2.28).

Finally, and in analogy to the effective coercivity constant defined in (2.11), a macroscopic one-cell coercivity constant $\widehat{B}$ is defined by

$$
\widehat{B}(\overline{\mathbf{F}}) \equiv \min _{\|\mathbf{a}\|=\|\mathbf{n}\|=1}\left\{a_{i} n_{j} \widehat{L}_{i j k l}(\overline{\mathbf{F}}) a_{k} n_{l}\right\} .
$$

With the definition of the three coercivity constants (also, and equivalently, termed stability constants), $\widehat{B}(\overline{\mathbf{F}}), \beta_{0}(\overline{\mathbf{F}})$ and $\beta(\overline{\mathbf{F}})$, for the macroscopic loading $\overline{\mathbf{F}}$, the stage has been set for discussing the stability of the periodic porous solid at that load level. It follows from the definitions of these three coercivity constants (see Geymonat et al., 1993) that the following relation holds for arbitrary vectors $\mathbf{a}$ and $\mathbf{n}$ :

$$
a_{i} n_{j} \widehat{L}_{i j k l}(\overline{\mathbf{F}}) a_{k} n_{l} \geqslant \beta_{0}(\overline{\mathbf{F}})\|\mathbf{a}\|^{2}\|\mathbf{n}\|^{2} \geqslant \beta(\overline{\mathbf{F}})\|\mathbf{a}\|^{2}\|\mathbf{n}\|^{2} \Longrightarrow \widehat{B}(\overline{\mathbf{F}}) \geqslant \beta_{0}(\overline{\mathbf{F}}) \geqslant \beta(\overline{\mathbf{F}}) .
$$

More specifically, the above relations indicate that when the one-cell based homogenized energy is the correct one (i.e., $\beta(\overline{\mathbf{F}})>0$ in which case $\widetilde{W}(\overline{\mathbf{F}})=\widehat{W}(\overline{\mathbf{F}})$ ), the homogenized energy function is strictly rank-one convex. Moreover, microscopic stability $(\beta(\overline{\mathbf{F}})>0$, which means from (2.28), that the solid is stable to bounded perturbations of arbitrary wavelength $\omega)$ implies macroscopic stability $(\widehat{B}(\overline{\mathbf{F}})>0$, which means that the corresponding one-cell based homogenized moduli $\widehat{\mathbf{L}}$ are also strongly elliptic).

Finding the domain in macroscopic strain $(\overline{\mathbf{F}})$ space for which the material is microscopically stable, i.e., $\beta(\overline{\mathbf{F}})>0$, although feasible thanks to (2.26), requires tedious and time consuming calculations since one has to scan using a fine grid the $(0,2 \pi) \times(0,2 \pi) \times$ $(0,2 \pi)$ domain in Fourier $(\omega)$ space. On the other hand, finding the larger domain in the same macroscopic strain $(\overline{\mathbf{F}})$ space for which the one-cell homogenized solid $\widehat{W}(\overline{\mathbf{F}})$ is macroscopically stable, i.e., $\widehat{B}(\overline{\mathbf{F}})>0$, is a rather straightforward calculation since it only requires the determination of the homogenized moduli $\widehat{\mathbf{L}}(\overline{\mathbf{F}})$ at each macroscopic deformation $\overline{\mathbf{F}}$. Calculating these two (i.e., the microscopic and macroscopic) stability domains for certain non-linear solids with different porous microstructures is the object of the present work.

An interesting observation about the loss of macroscopic stability is in order, before ending this subsection. It has been shown by Geymonat et al. (1993) that $\widehat{B}(\overline{\mathbf{F}})$ and $\beta_{0}(\overline{\mathbf{F}})$ always vanish simultaneously, i.e., if $\beta_{0}(\overline{\mathbf{F}})=0$, then it implies that $\widehat{B}(\overline{\mathbf{F}})=0$. This means from (2.30) that the onset of a long-wavelength instability $(\boldsymbol{\omega} \rightarrow \mathbf{0}$ - the wavelength of the eigenmode is much larger compared to the unit cell size) is always detectable as a loss of strong ellipticity of the one-cell homogenized moduli. Therefore, the following remark can be made about the first - in a monotonic loading process which will be defined subsequently-loss of microscopic stability $\left(\beta\left(\overline{\mathbf{F}}_{\mathrm{c}}\right)=0\right)$ in a microstructured elastic solid at some critical macroscopic deformation $\overline{\mathbf{F}}_{\mathrm{c}}$ : if $\beta_{0}\left(\overline{\mathbf{F}}_{\mathrm{c}}\right)=\beta\left(\overline{\mathbf{F}}_{\mathrm{c}}\right)$, the wavelength of the first instability encountered is much larger than the unit cell size and hence the instability can be detected as a loss of strong ellipticity of the one-cell homogenized moduli $\widehat{\mathbf{L}}$ since $\widehat{B}\left(\overline{\mathbf{F}}_{\mathrm{c}}\right)=0$. For the case when $\beta_{0}\left(\overline{\mathbf{F}}_{\mathrm{c}}\right)>\beta\left(\overline{\mathbf{F}}_{\mathrm{c}}\right)=0$, the first instability encountered in the 
loading process has a finite wavelength, and from that point on the one-cell homogenization is no longer physically meaningful and hence $\widehat{W}$ cannot provide any useful information about the solid. Henceforth, a tedious numerical process that follows the bifurcated equilibrium solutions is required to determine the response of the solid under the macroscopic strains in the neighborhood of $\overline{\mathbf{F}}_{\mathrm{c}}$ and beyond.

\subsection{Onset-of-failure surfaces}

To summarize the discussion in the two previous subsections, the elastomer's macroscopic stability constant $B(\overline{\mathbf{F}})$ is defined for the different microgeometries as follows:

$$
B(\overline{\mathbf{F}}) \equiv \begin{cases}\widetilde{B}(\overline{\mathbf{F}}) \text { random } & \left(\widetilde{\mathbf{L}}(\overline{\mathbf{F}})=\partial^{2} \widetilde{W} / \partial \overline{\mathbf{F}} \partial \overline{\mathbf{F}} \text { SOH only }\right) \\ \widehat{B}(\overline{\mathbf{F}}) \text { periodic } & \left(\widehat{\mathbf{L}}(\overline{\mathbf{F}})=\mathbf{L}^{\mathrm{H}}(\overline{\mathbf{F}}) \text { for FEM }, \widehat{\mathbf{L}}(\overline{\mathbf{F}})=\partial^{2} \widehat{W} / \partial \overline{\mathbf{F}} \partial \overline{\mathbf{F}} \text { for SOH }\right)\end{cases}
$$

The top definition (see (2.11)) is employed for random microgeometries where a $\mathrm{SOH}$ approximation (explained in the next section) of the effective energy density $\widetilde{W}(\overline{\mathbf{F}})$ is used. The bottom definition (see (2.29)) is employed for periodic microgeometries, since a calculation of $\widetilde{W}(\overline{\mathbf{F}})$ is not feasible in this case. When an FEM approach (also explained in the next section) is used, the one-cell homogenized moduli are calculated using $\mathbf{L}^{\mathrm{H}}(\overline{\mathbf{F}})$ defined in (2.18), while for the $\mathrm{SOH}$ approximation the one-cell homogenized moduli are obtained by differentiating $\widehat{W}(\overline{\mathbf{F}})$ (see $\left.(2.16)_{1}\right)$.

For the periodic microgeometry, the microscopic stability constant $\beta(\overline{\mathbf{F}})$ is also defined according to (2.26). The stage has thus been set to introduce the corresponding (i) macroscopic and (ii) microscopic onset-of-failure surfaces in macroscopic load space. These surfaces are, respectively, defined as the regions in macroscopic load space where (i) $B>0$, outside which the solid is unstable since the homogenized energy density is no longer strongly elliptic, and (ii) $\beta>0$, inside which the infinite-cell periodic solid is stable and where the one-cell homogenization procedure is valid $\widehat{W}=\widetilde{W}$.

The parameterization of the loading path in deformation space is needed for the determination of the above-defined surfaces. A loading path $\overline{\mathbf{F}}(\lambda)$ is considered parameterized by a scalar quantity $\lambda \geqslant 0$, termed load parameter, starting at $\lambda=0$ for $\overline{\mathbf{F}}=\mathbf{I}$, that increases monotonically with increasing applied macroscopic load. In a physically meaningful problem the solid under investigation is stable in its undeformed, stress-free state, i.e., $B(\mathbf{I}) \geqslant \beta(\mathbf{I})>0$ (initially $\overline{\mathbf{F}}=\mathbf{I}$ ). The macroscopic onset-of-failure surface is thus defined by

$$
B\left(\lambda_{\mathrm{cM}}\right)=0, \quad B(\lambda)>0, \quad 0 \leqslant \lambda<\lambda_{\mathrm{cM}} ; \quad B(\lambda) \equiv B(\overline{\mathbf{F}}(\lambda)) .
$$

In other words $\lambda_{\mathrm{cM}}$ is the lowest root of $B(\lambda)$, the macroscopic stability constant defined in (2.11) and evaluated on the load path $\overline{\mathbf{F}}(\lambda)$. Similarly to (2.32), the microscopic onset-offailure surface is given by

$$
\beta\left(\lambda_{\mathrm{cm}}\right)=0, \quad \beta(\lambda)>0, \quad 0 \leqslant \lambda<\lambda_{\mathrm{cm}} ; \quad \beta(\lambda) \equiv \beta(\overline{\mathbf{F}}(\lambda)) .
$$

In other words $\lambda_{\mathrm{cm}}$ is the lowest root of $\beta(\lambda)$, the microscopic stability constant defined in (2.26) and evaluated on the load path $\overline{\mathbf{F}}(\lambda)$.

The above general definition of the onset-of-failure surfaces requires the selection of a loading path. For the plane strain problem of interest in this work, a proportional strain path in macroscopic logarithmic strain space will be subsequently specified. 


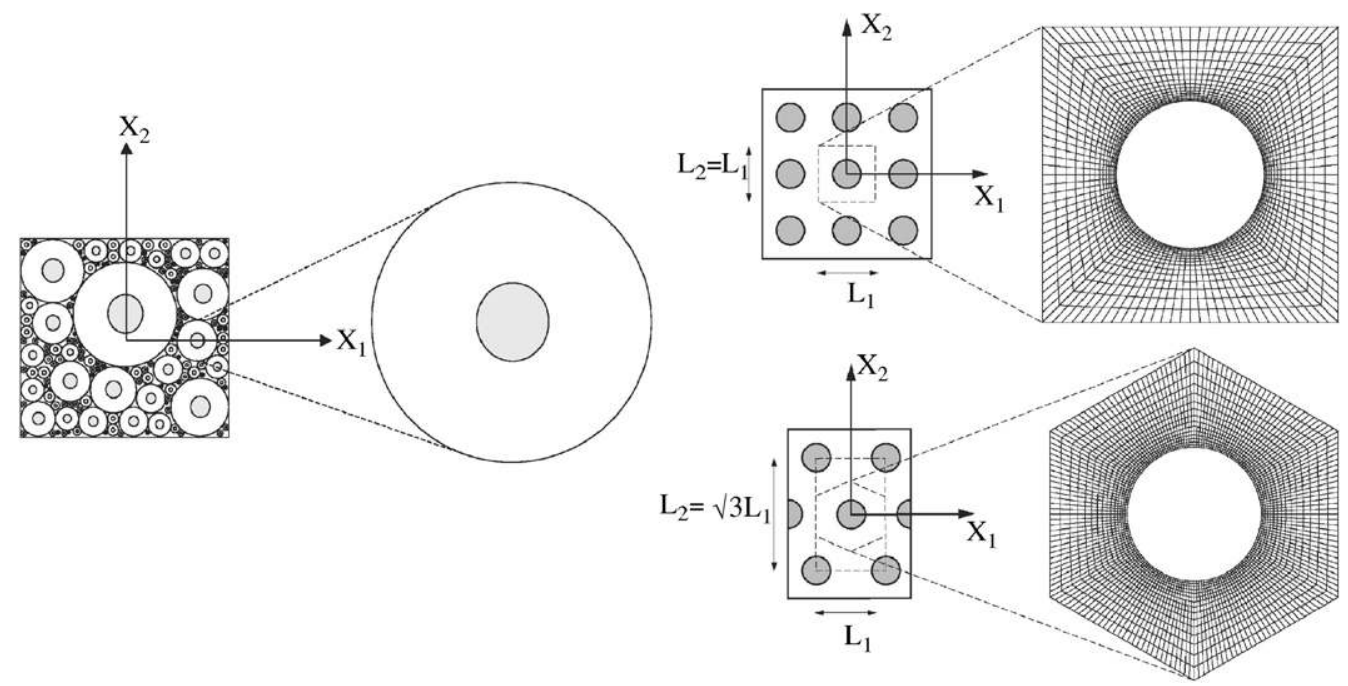

Fig. 1. Reference configuration depiction of the various microgeometries investigated: (a) random polydisperse and (b) perfect periodic with (i) square and (ii) hexagonal arrangement of circular voids.

\section{Calculation methods for plane-strain loading of elastomers with cylindrical pores}

The results presented thus far are valid for three-dimensional porous elastomers with arbitrary microgeometry, macroscopic loading and matrix constitutive laws. From this point on, attention is focused on plane-strain deformations (in the $X_{1}-X_{2}$ plane) of porous elastomers consisting of cylindrical voids perpendicular to the plane of deformation and aligned in the $X_{3}$ axis direction. The voids are taken to have initially circular cross section and initial volume fraction $f_{0}$. Two types of pore distributions (in the reference configuration) are considered: (a) statistically isotropic random and (b) periodic with (i) square and (ii) hexagonal arrangements as depicted in Fig. 1.

This section pertains to the load path description and to the calculation methods employed in this study. Following the description of the load paths adopted, the second subsection presents the second-order variational estimates used in deriving approximations for the effective properties of the porous elastomer. Within the same subsection are presented the estimates for the linear comparison composite as well as information required for the calculation of the microstructure evolution. Finally the third subsection presents the main assumptions used in the FEM calculations.

\subsection{Loading paths in plane strain}

Note that the applied macroscopic deformation $\overline{\mathbf{F}}$ in this context is entirely characterized by the four in-plane components: $\bar{F}_{11}, \bar{F}_{22}, \bar{F}_{12}, \bar{F}_{21}$, since the out-of-plane components are fixed: $\bar{F}_{13}=\bar{F}_{31}=\bar{F}_{23}=\bar{F}_{32}=0$, and $\bar{F}_{33}=1$. In this regard, it proves expedient to exploit the objectivity of $\widetilde{W}$, by ignoring macroscopic rigid rotations $(\overline{\mathbf{R}}=\mathbf{I})$, and to make use of the decomposition $U_{i j}=Q_{i k} D_{k l} Q_{j l}$, in order to express the (in-plane) macroscopic 
deformation gradient $\overline{\mathbf{F}}$ as ${ }^{4}$ :

$$
\bar{F}_{i j}=\bar{U}_{i j}=\left[\begin{array}{cc}
\cos \theta & -\sin \theta \\
\sin \theta & \cos \theta
\end{array}\right]\left[\begin{array}{cc}
\lambda_{1} & 0 \\
0 & \lambda_{2}
\end{array}\right]\left[\begin{array}{cc}
\cos \theta & \sin \theta \\
-\sin \theta & \cos \theta
\end{array}\right] .
$$

In the above expression, $\lambda_{1}$ and $\lambda_{2}$ denote the in-plane macroscopic principal stretches, and $\theta$ serves to characterize the orientation (in the anti-clockwise sense relative to the fixed laboratory frame of reference) of the macroscopic, in-plane, Lagrangian principal axes (i.e., the principal axes of $\overline{\mathbf{U}}$ ). For convenience, the coordinates $X_{i}$ defining the periodic elastomer's axes of orthotropy in the reference configuration are identified here with the fixed laboratory frame of reference. For the initially isotropic random microgeometry, all choices of the fixed laboratory frame of reference are equivalent. In the sequel, the components of any tensorial quantity will be referred to $X_{i}$.

Next, a load path needs to be selected and parameterized. Here, without loss of generality, attention is restricted to proportional straining paths in principal logarithmic strain space. More specifically, it is assumed that the ratio of the principal logarithmic strains $\varepsilon_{i}$ is fixed, namely:

$$
\ln \left(\lambda_{1}\right) \equiv \varepsilon_{1}=\lambda \cos \varphi, \quad \ln \left(\lambda_{2}\right) \equiv \varepsilon_{2}=\lambda \sin \varphi,
$$

where $\lambda$ is the monotonically increasing load parameter of the process and $\varphi$ is the load path angle. The microscopic and macroscopic onset-of-failure surfaces to be computed here are found by marching along (starting from $\lambda=0$ ) all radial paths $\varphi \in[0,2 \pi)$ in principal strain space for a fixed value of the principal axes orientation angle $\theta$.

\subsection{Second-order variational estimates}

As stated above, the determination via (2.8) of the effective stored-energy function $\widetilde{W}$ is a difficult problem, especially for porous elastomers with random microgeometry, where numerical approaches based on detailed microstructural models become infeasible. Here, use will be made of the SOH theory of Lopez-Pamies and Ponte Castañeda (2006b) to generate estimates for $\widetilde{W}$ and its derivatives for the porous elastomers considered in this work.

The key concept behind the SOH theory is the construction of suitable variational principles utilizing the idea of a linear comparison composite (LCC). This homogenization technique, which is exact to second order in the heterogeneity contrast, has the capability to incorporate statistical information about the microstructure beyond the volume fraction and can be applied to large classes of hyperelastic composites including reinforced and porous rubbers. A detailed derivation of the theory can be found in Lopez-Pamies and Ponte Castañeda (2006a). For brevity, here only the main results specialized to porous elastomers are presented. Thus, the second-order estimate for the effective stored-energy function of a porous elastomer is given by

$$
\widetilde{W}(\overline{\mathbf{F}})=\left(1-f_{0}\right)\left[W\left(\widehat{\mathbf{F}}^{(1)}\right)-S_{i j}(\overline{\mathbf{F}})\left(\widehat{F}_{i j}^{(1)}-\bar{F}_{i j}^{(1)}\right)\right],
$$

\footnotetext{
${ }^{4}$ Here and subsequently, Latin indices range from 1 to 2 .
} 
where $\mathbf{S}$ is the nominal stress in the matrix, introduced in (2.5). The variable $\widehat{\mathbf{F}}^{(1)}$ is an auxiliary deformation in the matrix (phase (1)) defined by the generalized secant condition:

$$
S_{i j}\left(\widehat{\mathbf{F}}^{(1)}\right)-S_{i j}(\overline{\mathbf{F}})=L_{0 i j k l}\left(\widehat{F}_{k l}^{(1)}-\bar{F}_{k l}\right),
$$

while $\overline{\mathbf{F}}^{(1)}$ is the average deformation in the matrix, which will be made explicit subsequently. Finally, $\mathbf{L}_{0}$ is a constant, fourth-order tensor with major symmetry (but with no other symmetries) that is identified with the modulus tensor of a fictitious linear thermoelastic material with a matrix stored-energy function:

$$
W_{\mathrm{T}}(\mathbf{F})=W(\overline{\mathbf{F}})+S_{i j}(\overline{\mathbf{F}})\left(F_{i j}-\bar{F}_{i j}\right)+\frac{1}{2}\left(F_{i j}-\bar{F}_{i j}\right) L_{0 i j k l}\left(F_{k l}-\bar{F}_{k l}\right) .
$$

The relevant LCC is then defined as a two-phase material with a vacuous inclusion phase and a linear thermoelastic matrix phase with stored-energy function (3.5), and precisely the same microstructure as the actual non-linear porous material. The corresponding overall stored-energy function of the LCC is given by (Laws, 1973)

$$
\widetilde{W}_{\mathrm{T}}(\overline{\mathbf{F}})=\left(1-f_{0}\right) W(\overline{\mathbf{F}})+\frac{1}{2} S_{i j}(\overline{\mathbf{F}}) L_{0 i j k l}^{-1}\left[\widetilde{L}_{0 k l m n}-\left(1-f_{0}\right) L_{0 k l m n}\right] L_{0 m n p q}^{-1} S_{p q}(\overline{\mathbf{F}}),
$$

where $\widetilde{\mathbf{L}}_{0}$ is the effective modulus tensor of the LCC, to be subsequently detailed.

Having characterized the local and overall behavior of the pertinent LCC, attention is turned next to the prescriptions for the $\overline{\mathbf{F}}^{(1)}$ and the LCC moduli $\mathbf{L}_{0}$ of the matrix phase, introduced in (3.3) and (3.4). These quantities are determined using the phase average $\langle\mathbf{F}\rangle^{(1)}$ and the covariance tensor

$$
C_{\mathbf{F}}^{(1)}{ }_{i j k l} \equiv\left\langle\left(F_{i j}-\bar{F}_{i j}^{(1)}\right)\left(F_{k l}-\bar{F}_{k l}^{(1)}\right)\right\rangle^{(1)},
$$

of the deformation fields in the matrix phase of the LCC. More specifically, $\overline{\mathbf{F}}^{(1)}$ is given explicitly in terms of the effective modulus tensor $\widetilde{\mathbf{L}}_{0}$ via

$$
\bar{F}_{i j}^{(1)}=\bar{F}_{i j}+\frac{1}{1-f_{0}} L_{0 i j k l}^{-1}\left(\widetilde{L}_{0 k l m n}-\left(1-f_{0}\right) L_{0 k l m n}\right) L_{0 m n p q}^{-1} S_{p q}(\overline{\mathbf{F}}) .
$$

On the other hand, $\mathbf{L}_{0}$ is obtained from certain optimality conditions (given further below) involving the field fluctuations in the LCC. For isotropic matrix phases, the tensor $\mathbf{L}_{0}$ should be taken of the form (see Lopez-Pamies and Ponte Castañeda, 2006b):

$$
L_{0 i j k l}=\bar{Q}_{r m} \bar{Q}_{j n} \bar{Q}_{s p} \bar{Q}_{l q} \bar{R}_{i r} \bar{R}_{k s} L_{m n p q}^{*},
$$

where $\mathbf{L}^{*}$ is orthotropic with respect to the $X_{i}$ axes, with non-zero components $L_{111}^{*}=\ell_{1}^{*}$, $L_{2222}^{*}=\ell_{2}^{*}, L_{1212}^{*}=\ell_{3}^{*}$, and $L_{1122}^{*}=\ell_{4}^{*}$ and where:

$$
L_{2121}^{*}=\ell_{3}^{*} \quad \text { and } \quad L_{1221}^{*}=\sqrt{\left(\ell_{1}^{*}-\ell_{3}^{*}\right)\left(\ell_{2}^{*}-\ell_{3}^{*}\right)}-\ell_{4}^{*} .
$$

Note that, since $\overline{\mathbf{Q}}$ and $\overline{\mathbf{R}}$ can be readily computed from the applied loading $\overline{\mathbf{F}}$, it is inferred from condition (3.10) that the modulus tensor $\mathbf{L}_{0}$ of the matrix phase in the LCC contains four independent unknowns: $\ell_{1}^{*}, \ell_{2}^{*}, \ell_{3}^{*}$, and $\ell_{4}^{*}$. The optimal value of these parameters is determined by the relations:

$$
\left(\widehat{F}_{i j}^{(1)}-\bar{F}_{i j}\right) \frac{\partial L_{0 i j k l}}{\partial \ell_{\alpha}^{*}}\left(\widehat{F}_{k l}^{(1)}-\bar{F}_{k l}\right)=\frac{2}{1-f_{0}} \frac{\partial \widetilde{W}_{\mathrm{T}}}{\partial \ell_{\alpha}^{*}} \quad(\alpha=1,2,3,4) .
$$

Note that the RHS of this equation can be related to certain traces of the covariance tensor $\mathbf{C}_{\mathbf{F}}^{(1)}$ defined in (3.7), thus providing a physical meaning to the auxiliary deformation $\widehat{\mathbf{F}}^{(1)}$. 
In summary, Eqs. (3.4) and (3.11) constitute a closed system of eight coupled, nonlinear, algebraic equations for the eight unknowns formed by the four components of $\widehat{\mathbf{F}}^{(1)}$ and the four independent components of $\mathbf{L}_{0}$ (denoted by $\ell_{\alpha}^{*}$ ). It is possible (see LopezPamies and Ponte Castañeda, 2006b) to solve in closed-form Eqs. (3.11) for the four components of $\widehat{\mathbf{F}}^{(1)}$ in terms of the parameters $\ell_{\alpha}^{*}$. The resulting expressions can then be substituted into Eq. (3.4) to obtain a system of four equations for the four unknowns $\ell_{\alpha}^{*}$ $(\alpha=1,2,3,4)$, which must be solved numerically.

Having computed all the values of $\ell_{\alpha}^{*}$ for a given porosity, material and loading, the values of the components of $\overline{\mathbf{F}}^{(1)}$ and $\widehat{\mathbf{F}}^{(1)}$ can be readily determined from (3.8) and (3.11), respectively. In turn, the second-order estimate for the effective behavior of porous elastomers can be computed, from relation (3.3), using these results. Finally, having determined $\widetilde{W}(\overline{\mathbf{F}})$ via (3.3), it is straightforward to compute its second derivative, i.e., the macroscopic elasticity tensor $\widetilde{\mathbf{L}}(\overline{\mathbf{F}})$ according to (2.10), in order to examine the macroscopic stability of the solid via the coercivity constant $\widetilde{B}(\overline{\mathbf{F}})$ defined in (2.11).

\subsubsection{Estimates for the linear comparison composite}

At this stage, the only variable that remains to be specified is the effective modulus tensor $\widetilde{\mathbf{L}}_{0}$ of the LCC. In view of the particulate type of microstructures of interest in this work, it is appropriate to make use of the Hashin-Shtrikman $(\mathrm{H}-\mathrm{S})$ estimate (not to be confused with the Hashin-Shtrikman bounds) for the effective modulus tensor, given by the formula

$$
\widetilde{\mathbf{L}}_{0}=\mathbf{L}_{0}+f_{0}\left[\left(1-f_{0}\right) \mathbf{P}-\mathbf{L}_{0}^{-1}\right]^{-1} .
$$

Here, $\mathbf{P}$ is a microstructural tensor that depends on the size, shape and orientation of the pores, as well as on their spatial distribution in the reference configuration. In particular, the tensor $\mathbf{P}$ depends on whether the distribution of the pores is periodic (Nemat-Nasser et al., 1982; Suquet, 1990a, b), or random (Willis, 1977; Ponte Castañeda and Willis, 1995). The explicit expressions for $\mathbf{P}$ for the three types of microstructures considered in this work are given in Appendix B. It is important to recall that these estimates of the H-S-type are known to be accurate for small to moderate initial porosities and that they become inaccurate for large porosities, near the percolation limit. However, it should be emphasized that the second-order estimates (3.3) can still be used beyond this range, provided that a more sophisticated estimate is adopted for the LCC.

\subsubsection{Microstructure evolution}

Generally speaking, the problem of characterizing the evolution of the microstructure in composites is exceedingly difficult, due to the large number of microstructural variables involved. However, for the type of elastomers with particulate microstructures considered here, it is possible (see Section 5 in Lopez-Pamies and Ponte Castañeda, 2006a) to identify and characterize the evolution of suitable microstructural variables.

The evolution of the size, shape, and orientation of the pores are governed-on average - by the average deformation gradient in the porous phase $\overline{\mathbf{F}}^{(2)}$. Thus, the relevant microstructural variables characterizing the size, shape, and orientation of the pores, identified here as the volume fraction, $f$, the average aspect ratio, $a$, and the average orientation of the pores, $\alpha$, are determined by $\overline{\mathbf{F}}^{(2)}$. It is important to note that within the context of the second-order estimates (3.3), with the H-S-type approximation (3.12) for the effective behavior of the associated LCC, the deformation gradient field inside the pores 
turns out to be constant, i.e., $\mathbf{F}(\mathbf{X})=\overline{\mathbf{F}}^{(2)}$ for $\mathbf{X} \in \Omega_{0}^{(2)}$. As a result, a circular pore of radius $R^{i}$ and centered at $\mathbf{X}^{i}$ in the undeformed configuration, defined by

$$
E_{0}^{i}=\left\{\mathbf{X} \mid\left\|\mathbf{X}-\mathbf{x}^{i}\right\| \leqslant R^{i}\right\},
$$

will deform according to $x_{k}-x_{k}^{i}=\bar{F}_{k l}^{(2)}\left(X_{l}-X_{l}^{i}\right)$, with $\mathbf{x}^{i}$ denoting the center of the pore in the deformed configuration. Thus, the circular pore defined by (3.13) evolves into the ellipse:

$$
E^{i}=\left\{\mathbf{x} \mid\left\|\left(x_{k}-x_{k}^{i}\right) Z_{k l}\right\| \leqslant R^{i}\right\},
$$

in the deformed configuration, where $\mathbf{Z}=\left(\overline{\mathbf{F}}^{(2)}\right)^{-1}$. The eigenvalues $1 / z_{1}^{2}$ and $1 / z_{2}^{2}$ of the symmetric second-order tensor $Z_{k i} Z_{k j}$ define the current aspect ratio $a=z_{2} / z_{1}$ of the pore in the deformed configuration. Hence, the principal directions of $Z_{k i} Z_{k j}$, denoted here by the rectangular Cartesian axes $X_{i}^{\prime}$, are the principal semi-axes of the elliptical pore in the deformed configuration. Note that the orientation of $X_{i}^{\prime}$ relative to $X_{i}$ is entirely described by the angle $\alpha$ (measured counterclockwise). Moreover, by making use of the uniformity of the deformation in the vacuous phase, the current volume fraction of the pores in the deformed configuration may be simply obtained via

$$
f=\frac{\operatorname{det} \overline{\mathbf{F}}^{(2)}}{\operatorname{det} \overline{\mathbf{F}}} f_{0} .
$$

In short, the evolution of the size, shape, and orientation of the pores is completely characterized by $\overline{\mathbf{F}}^{(2)}$, via expressions (3.14) and (3.15), which can be readily computed by making use of the overall condition $\overline{\mathbf{F}}=\left(1-f_{0}\right) \overline{\mathbf{F}}^{(1)}+f_{0} \overline{\mathbf{F}}^{(2)}$, together with estimate (3.8) for the average deformation gradient $\overline{\mathbf{F}}^{(1)}$ in the matrix phase of the LCC.

Finally some comments are in order concerning the evolution of the distribution of the pores (i.e., the relative motion of the center of the underlying vacuous inclusions) as a function of the applied deformation $\overline{\mathbf{F}}$. For the simple periodic (square and hexagonal) microgeometries of interest in this work (Fig. 1b), it can be shown rigorously that the relative motion of the centers of the underlying pores are governed by the macroscopic deformation gradient $\overline{\mathbf{F}}$ (and not by the average field $\overline{\mathbf{F}}^{(2)}$ in the vacuous phase), at least up to the onset of the first microscopic instability. On the other hand, for porous elastomers with the random microgeometry (Fig. 1a), the situation is less clear. Indeed, the authors are not aware of any rigorous result regarding the evolution of the distribution of the underlying pores in elastomers with this type of microstructure. However, it has been proposed (Lopez-Pamies and Ponte Castañeda, 2006a) as an approximation that in this case the voids also move, on the average, with the macroscopic flow, as determined by $\overline{\mathbf{F}}$. Accordingly, for all three microgeometries studied in this paper, the evolution of the distribution of the pores is taken to be controlled by the macroscopic deformation gradient $\overline{\mathbf{F}}$. In particular, this implies that a pore centered at $\mathbf{X}^{i}$ in the undeformed configuration will move according to $x_{k}^{i}=\bar{F}_{k l} X_{l}^{i}$.

\subsection{Finite element method}

In addition to the above-described, approximate, SOH-based method, a more accurate FEM approach is also employed to calculate the deformed configuration (i.e., to find $\mathbf{u}_{\bar{F}}^{\prime}$ ), the one-cell homogenized moduli $\widehat{\mathbf{L}}$, as well as the microscopic $\lambda_{\mathrm{cm}}$ and macroscopic $\lambda_{\mathrm{cM}}$ onset-of-failure loads for the periodic elastomers. The details of the corresponding FEM 
algorithm are given in Triantafyllidis et al. (2006). However, for reasons of completeness of the presentation, a brief description of this algorithm is included here.

All FEM calculations use four node (8 d.o.f.) isoparametric, bilinear, quadrilateral elements with a $2 \times 2$ Gaussian integration scheme. For the one-cell square microgeometries (see Fig. 1b) the mesh has 1800 elements with 1920 nodes (3840 d.o.f). For the one-cell hexagonal microgeometries (see Fig. 1b) a 2700 mesh with 2880 nodes (5760 d.o.f.) is used. For the imperfect $4 \times 4$ cell aggregate (see Fig. 8) the mesh has 28,800 elements with 29,985 nodes $(59,970$ d.o.f.). This discretization is found to be more than adequate for the accuracy of the numerical calculations, since further mesh refinement does not result in appreciably different onset-of-failure curves.

The governing equations for the unit-cell deformation problem (2.14) subjected to $\mathbf{F}(\lambda)$, defined in (3.1), are solved using an incremental Newton-Raphson algorithm. The typical step size $\Delta \lambda$ of the load parameter $\lambda$, defined in (3.2), is $\Delta \lambda=10^{-3}$ for biaxial compression paths and $\Delta \lambda=10^{-2}$ for all other paths. Occasionally larger step sizes are employed to shorten calculation time. For most cases three iterations are required for convergence at each load step and the accuracy criterion required to stop the iterations, based on the nominal stress $(\mathbf{S}=\partial W / \partial \mathbf{F})$ vector's Euclidean norm, is $\|\mathbf{S}\| \leqslant 10^{-4}\|\overline{\mathbf{S}}\|$. The graphs of the microscopic and macroscopic onset-of-failure surfaces are calculated using 360 different load path angles (staring with $\varphi=0$ and repeating the calculations by increasing each time the path angle by $\Delta \varphi=\pi / 180$ ).

Calculations, at each load $\lambda$, of the one-cell homogenized moduli tensor $\widehat{\mathbf{L}}(\lambda)\left(=\mathbf{L}^{\mathrm{H}}(\lambda)\right)$ are based on (2.18), (2.19) as detailed in Triantafyllidis et al. (2006). The thus calculated tensor $\widehat{\mathbf{L}}(\lambda)$ is then used to find the macroscopic coercivity constant $\widehat{B}(\overline{\mathbf{F}}(\lambda))$ defined in (2.29) and subsequently the macroscopic onset-of-failure load $\lambda_{\mathrm{cM}}$ according to definition (2.32). The calculation of the microscopic onset-of-failure load $\lambda_{\mathrm{cm}}$, defined in (2.33), is based on the macroscopic coercivity constant $\beta(\overline{\mathbf{F}}(\lambda))$. This calculation in turn requires according to (2.26) knowledge of the corresponding critical wavenumber $\omega$.

In view of the corresponding time-consuming computations, full Bloch-wave calculations are done for only one periodic elastomer which has a square microgeometry, an initial porosity $f_{0}=\pi / 16$ and a neo-Hookean matrix, for a relatively coarse mesh of the unit cell by using the algorithm introduced by Triantafyllidis et al. (2006). For this solidand for macroscopic strains aligned with the axes of orthotropy $(\theta=0)$ - the critical wavenumbers are found to be either $\omega_{1} L_{1}=\omega_{2} L_{2}=\pi$ or $\omega_{1} L_{1}, \omega_{2} L_{2} \rightarrow 0$, depending on the load path angle $\varphi$. Having thus established that for this periodic elastomer the only microscopic bifurcation modes are periodic on a $\mathbf{k} D$ super-cell with $\mathbf{k}=(2,2)$ (see discussion following (2.22)), a $2 \times 2$ cell aggregate with the final refined mesh and periodic boundary conditions is used to capture more accurately the strains at the onset of the first bifurcation.

\section{Application to specific materials}

This section presents the calculations for porous elastomers with different matrix properties and microgeometries. Following the description of the different matrix constitutive laws used in the calculations, the results are organized in three groups as follows: the first group, Figs. 2, 3, pertains to the influence of microgeometry and matrix constitutive law on the stress-strain response and on the porosity/aspect ratio evolution of porous hyperelastic solids under equibiaxial and uniaxial loading. The second group, 
Figs. 4-10, investigates the influence of initial porosity and microgeometry on the microscopic and macroscopic onset-of-failure curves in neo-Hookean matrix porous elastomers under plane strain loading with principal directions aligned with the axes of orthotropy of the elastomer. Finally the third group, Figs. 11 and 12, addresses the influence of matrix material and loading axes orientation on the failure surfaces of porous elastomers for a fixed initial porosity.

An important general remark about the onset-of-failure surfaces is in order at this point. For the calculation of the first - as the load parameter $\lambda$ increases along a given load path $\overline{\mathbf{F}}(\lambda)$-microscopic (at $\lambda_{\mathrm{cm}}$ ) or macroscopic (at $\lambda_{\mathrm{cM}}$ ) instability to be physically meaningful, one must ensure that no other type of failure is encountered along the load path in question for loads lower than $\lambda_{\mathrm{cm}}$ (or lower than $\lambda_{\mathrm{cM}}$ in the case that the lower microscopic instability load $\lambda_{\mathrm{cm}}$ is not available). However, there are several phenomena that can signal some other (than loss of uniqueness or of rank-one convexity) type of failure of the porous elastomer. These phenomena are (a) the instability that appears at large compressive strains on the free surface of the voids, (b) percolation, i.e., contact of adjacent pores leading to percolating network of voids, (c) strain lock-up, i.e., reaching of a maximum strain at the surface of the pores, which results in unrealistically stiff macroscopic response, and (d) pore self-contact (for the periodic case) or pore closure (for random microstructures), which dramatically alters the nature of the porous elastomer. Their detailed discussion is presented in Appendix A.

\subsection{Matrix material properties}

Two different hyperelastic, rank-one convex matrix materials are used here. They are distinguished by the response in simple shear, the first being linear the other reaching an asymptote at a finite strain. Their energy densities are given in terms of the following two invariants associated with $\mathbf{F}$ :

$$
I \equiv F_{i j} F_{i j}, \quad J \equiv \operatorname{det}\left(F_{i j}\right) .
$$

The first material is a compressible neo-Hookean solid, with strain energy $W(\mathbf{F})$ in plane strain:

$$
W=\frac{\mu}{2}[(I-2)-2 \ln J]+\frac{\kappa-\mu}{2}(J-1)^{2},
$$

where $\mu$ and $\kappa$ are, respectively, the shear and bulk moduli of the solid at zero strain. The response of this solid in simple shear is linear, with a unit slope because of the adopted non-dimensionalization for the stress.

The second material used is a compressible Gent solid, with a strain energy $W(\mathbf{F})$ in plane strain given by

$$
W=-\frac{\mu}{2}\left[J_{m} \ln \left(1-\frac{I-2}{J_{m}}\right)+2 \ln J\right]+\left[\frac{\kappa-\mu}{2}-\frac{\mu}{J_{m}}\right](J-1)^{2},
$$

where $\mu$ and $\kappa$ have the same interpretation as in (4.2) and $J_{m}$ is a constant related to the solid's strain saturation. Indeed, as expected from (4.3), the stresses become infinite as the strains (measured by the first invariant) approach $J_{m}+2$. This asymptotic behavior is motivated by the reversible elastic range response of natural rubbers which cannot sustain strains above a certain level without failure. The numerical value $J_{m}=50$, which has been used by Lopez-Pamies and Ponte Castañeda (2004b) is also adopted for all the numerical 
calculations reported in this work. Note that at the limit $J_{m} \rightarrow \infty$, the Gent energy density in (4.3) converges to the neo-Hookean energy density in (4.2).

Both neo-Hookean and Gent solids (for $\mu>0, J_{m}>0, \kappa>\left[\left(J_{m}+2\right) / J_{m}\right] \mu$ ) are polyconvex, as defined in Ball (1977), since they are convex functions of $I$ and $J$ for an isotropic solid and hence are rank-one convex, i.e., they must satisfy (2.7). Finally, it must be pointed out that upon a linearization of the deformation gradient, the above two laws reduce to the same small-strain linearly elastic solid with shear and bulk moduli $\mu$ and $\kappa$, respectively.

\subsection{Macroscopic stress-strain response and porosity/aspect ratio evolution}

Some general remarks applicable to all the results in this subsection (Figs. 2, 3) are first in order: all elastomers have the same initial porosity $\left(f_{0}=\pi / 16 \approx 0.2\right)$ and the same material properties at zero strain, since the two different matrix laws used have the same initial shear and bulk moduli $(\kappa / \mu=10)$. The calculations are done for two different initial microgeometries: (i) hexagonal and (ii) random polydisperse distributions of circular voids. The results for the periodic microgeometry are based on FEM (dotted lines) calculations, while for random microgeometry (dashed-dotted lines) SOH calculations are considered. All the stress-strain, porosity and aspect ratio evolution curves end at the loss of ellipticity of the corresponding homogenized moduli.

The influence of the matrix constitutive law on the macroscopic dimensionless Cauchy stress $\left(\sigma_{1} / \mu\right)$-logarithmic strain $\left(\varepsilon_{1}\right)$ response of porous elastomers, subjected to plane strain equibiaxial $\left(\varepsilon_{1}=\varepsilon_{2}\right)$ and uniaxial $\left(\varepsilon_{2}=0\right)$ loading is presented in Fig. 2 . Due to the decreasing porosity with increasing strain in compression, the different microgeometry

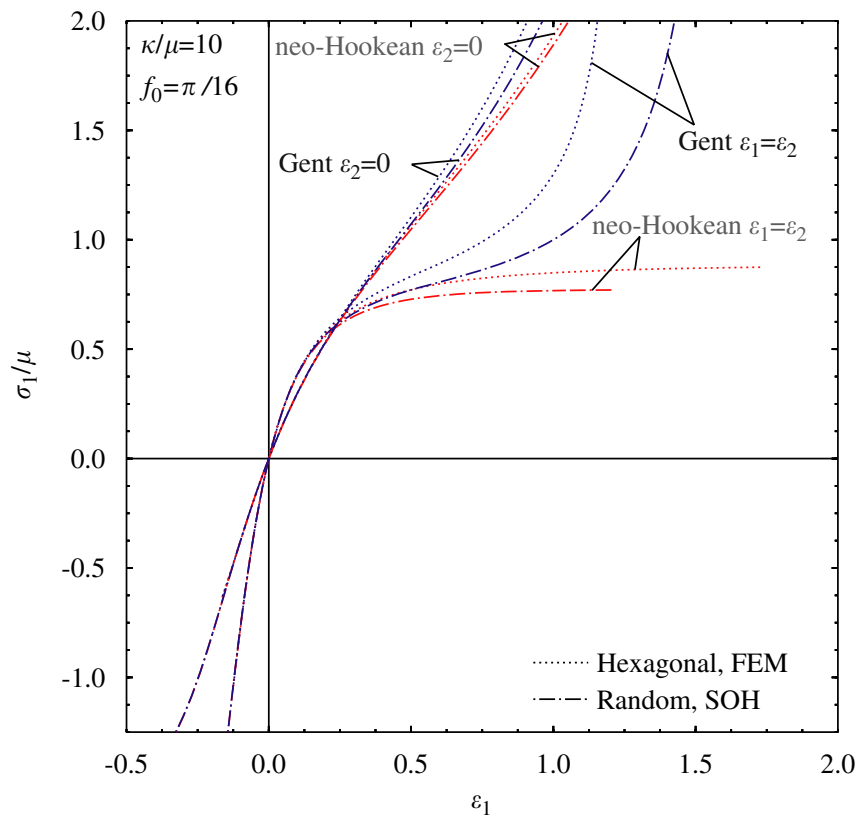

Fig. 2. Influence of matrix constitutive law on the macroscopic dimensionless Cauchy stress-logarithmic strain response of fixed initial porosity elastomers with (i) hexagonal and (ii) random polydisperse microgeometries under plane strain equibiaxial $\left(\varepsilon_{1}=\varepsilon_{2}\right)$ and uniaxial $\left(\varepsilon_{2}=0\right)$ loading. 
stress-strain curves for each loading path are almost indistinguishable from each other (at the scale plotted). For tension, and for macroscopic strains above $10 \%$ for the balanced biaxial loading (or above $20 \%$ for the uniaxial strain loading), the matrix constitutive law has a significant impact on the porous elastomer's response.

There are two competing mechanisms working in equibiaxial tension: the constitutive stiffening of the matrix material with increasing strains and the geometric softening due to increasing porosity. For the neo-Hookean matrix the two mechanisms balance out leading to a plateau in the macroscopic stress. For the Gent matrix the constitutive stiffening wins, resulting in a strain saturation (locking) of the porous elastomer, according to the results in Fig. 2.

To the two competing mechanisms (matrix stiffening due to material constitutive response and geometric softening due to the porosity increase) considered in explaining the biaxial loading in tension, one has to add for the uniaxial strain loading case the stiffening of the porous elastomer due to void ovalization. As a result, the stress-strain responses in uniaxial strain tension (compression) for all elastomers are stiffer (softer) than in the corresponding plane strain equibiaxial tension (compression) case.

The porosity $(f)$ evolution of the porous elastomer subjected to the two loading paths considered in Fig. 2 as function of the macroscopic strain $\left(\varepsilon_{1}\right)$ is depicted in Fig. 3a. Unlike the stress-strain results of Fig. 2, the porosity evolution under equibiaxial or uniaxial strain tension is remarkably insensitive to the microgeometry even for large tensile strains. As expected, the porosity is a monotonically increasing function for a wide range of macroscopic strains. Notice, however, that for the Gent material matrix, the porosity evolution curves show a sharp decline, following the reaching of a maximum value for admittedly large strains. This interesting behavior is due to the locking of the matrix material at the surface of the void. This implies that no further increase of the void size is possible with increasing macroscopic strain, and hence further macroscopic straining can

a

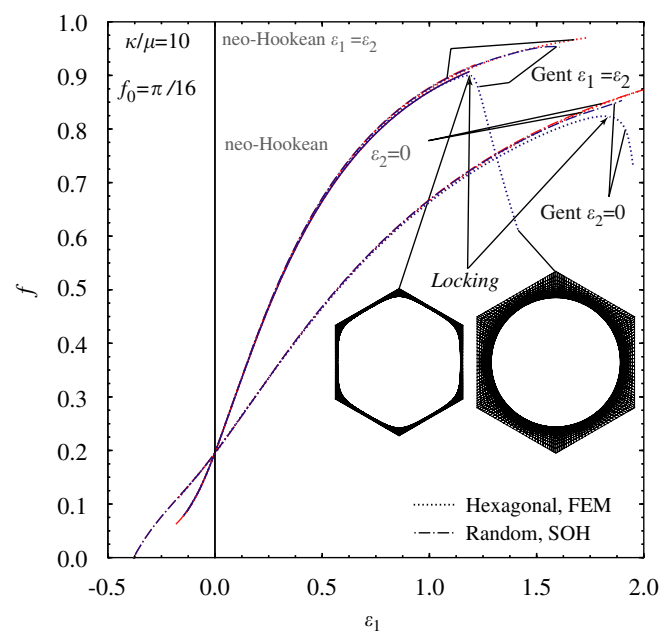

b

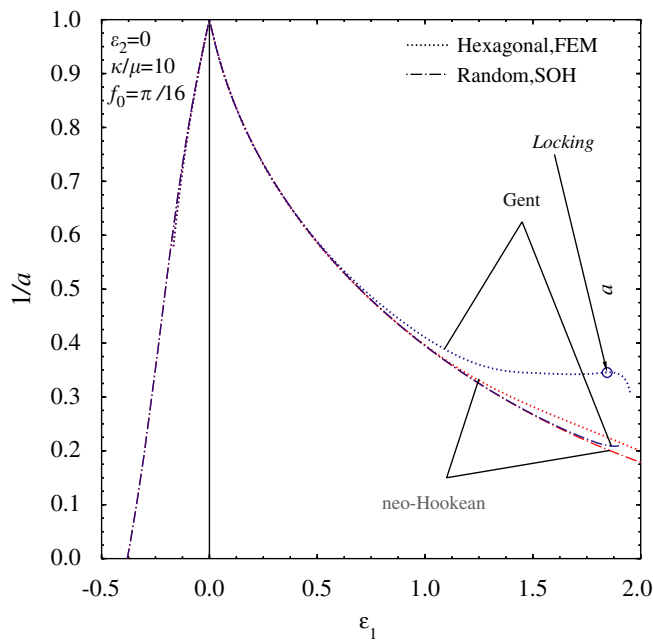

Fig. 3. Influence of matrix constitutive law on the porosity (a) and on the pore aspect ratio (b) evolution of fixed initial porosity $f_{0}=\pi / 16(\approx 0.2)$ elastomers with (i) hexagonal and (ii) random polydisperse microgeometries under plane strain equibiaxial $\left(\varepsilon_{1}=\varepsilon_{2}\right)$ and uniaxial $\left(\varepsilon_{2}=0\right)$ loading. Notice the locking occurring for the periodic Gent elastomer. 
only be accommodated by swelling of the matrix, exactly as seen by the two deformed configurations of the unit cell at maximum porosity strain and at a higher strain levelshown for the equibiaxial loading case-in Fig. 3a.

The aspect ratio evolution of the same elastomer under uniaxial plane strain is presented in Fig. 3b. As expected from the porosity reduction in compression, the aspect ratio evolution is practically independent of the microgeometry for compressive strains at the scale plotted. Notice, however, that noticeable discrepancies between the periodic and random microgeometries in the tensile region appear for strains about $\varepsilon_{1} \approx 1.25$ for the neo-Hookean matrix and about $\varepsilon_{1} \approx 0.75$ for the Gent matrix.

\subsection{Onset-of-failure curves for neo-Hookean solids}

The influence of porosity and microgeometry on the microscopic and macroscopic onset-of-failure curves in porous elastomers subjected to plane strain loading with principal directions aligned with the axes of orthotropy of the porous elastomer is presented in the second subsection, Figs. 4-10. All results in this subsection are calculated using a neo-Hookean matrix material with $\kappa / \mu=10$.

The onset-of-failure curves for porous elastomers with an initial porosity $f_{0}=\pi / 16 \approx$ 0.2 and an initially perfect square arrangement of circular voids are presented in Fig. 4 . In Fig. 4a are plotted, in principal logarithmic strain space, the microscopic (first bifurcation, in dotted lines) and macroscopic (loss of ellipticity of the homogenized moduli, in solid lines) onset-of-failure curves while in Fig. $4 \mathrm{~b}$ is plotted a blow-up of the biaxial

a

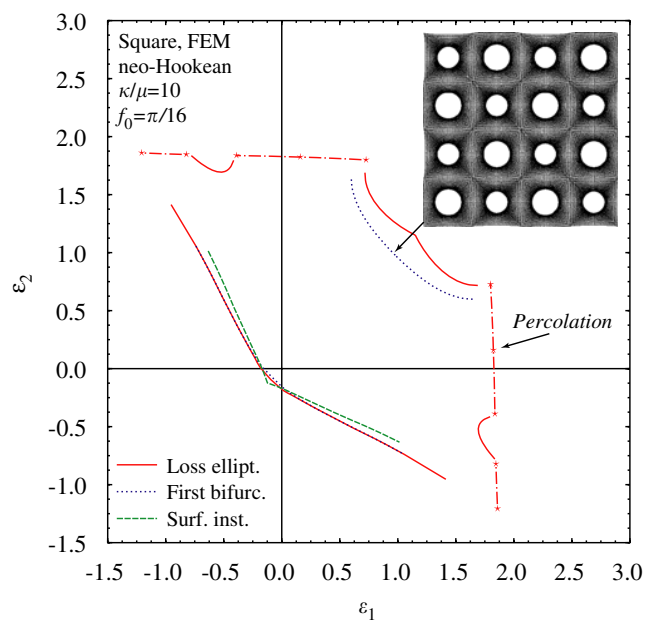

b

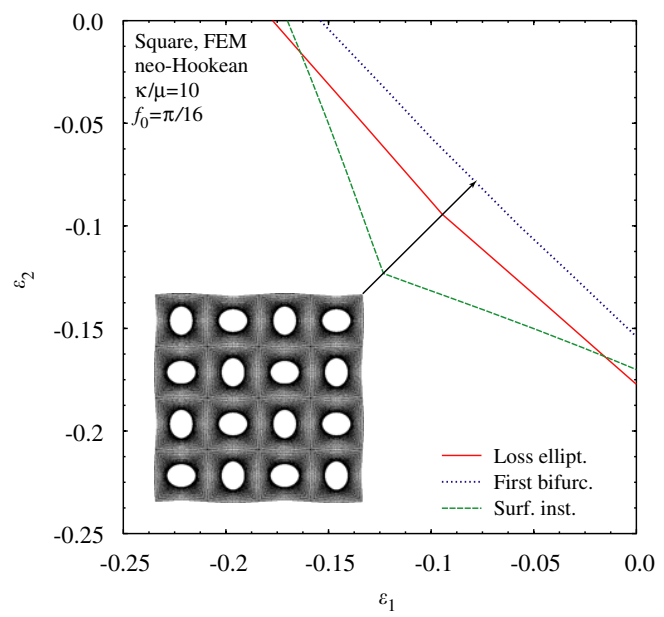

Fig. 4. In (a) are plotted in principal logarithmic strain space the microscopic (dotted lines) and macroscopic (solid lines) onset-of-failure curves of a neo-Hookean matrix elastomer with square microgeometry. The macroscopic strains corresponding to the onset of an instability at the free surface of the voids (dashed lines) and to percolation (dashed-dotted-starred lines) are also recorded. A blow-up of the biaxial compression region in (a) is plotted in (b). The eigenmodes corresponding to the first bifurcation encountered under plane strain equibiaxial tension and compression $\left(\varepsilon_{1}=\varepsilon_{2} \leqslant 0\right)$ are also depicted. 
compression region of Fig. 4a. The macroscopic strains corresponding to the onset of an instability at the free surface of the voids (dashed lines) and to percolation (dashed-dotted-starred lines) are also recorded. All results shown in this figure are based on FEM calculations. The first bifurcation curves are obtained by investigating the stability of a $2 \times 2$ cell aggregate subjected to periodic boundary conditions. Independent calculations for this porous elastomer, using Bloch-wave theory on one cell, (unpublished work, based on methodology and code described in Triantafyllidis et al. (2006)) have established that the only finite wavelength mode encountered at the onset of a bifurcation in this porous elastomer always corresponds to an antisymmetric mode, thus requiring calculations on a $2 \times 2$ cell aggregate subjected to periodic boundary conditions. The larger aggregate used in the FEM calculations for the equibiaxial case has the advantage of a better visualization of the eigenmode corresponding to the first bifurcation, as seen in the two insets of Fig. 4.

A bifurcation with a finite wavelength eigenmode is the first instability encountered under biaxial compression and biaxial tension, as seen in Fig. 4. The loss of ellipticity of the homogenized moduli (which corresponds to a bifurcation with an infinitely long wavelength mode) occurs at higher strains along the same path. For parts of the mixed loading region $\left(\varepsilon_{1} \varepsilon_{2}<0\right)$ the first instability encountered is a long wavelength instability and hence the microscopic and macroscopic onset-of-failure curves coincide. Observe that in the biaxial compression region, the first instability always occurs for macroscopic strains lower to the ones corresponding to the appearance of a surface instability at the void, while in part of the mixed loading region, the surface instability appears prior to any other failure (microscopic or macroscopic). Notice that for the remaining loading paths for which no instability of any type is found, the pore size of the porous elastomer increases to the point of percolation, i.e., adjacent voids come to contact as defined in Appendix A.

The eigenmodes corresponding to the first instability under equibiaxial tension $\left(\varepsilon_{1}=\right.$ $\left.\varepsilon_{2}>0\right)$ and equibiaxial compression $\left(\varepsilon_{1}=\varepsilon_{2}<0\right)$ are depicted, respectively, in Fig. 4a and b. As previously discussed, the critical mode is periodic on a $2 \times 2$ cell aggregate, thus explaining the repetitive pattern shown in Fig. 4. Notice that in the tensile case, the bifurcated mode consists of alternative change of the rate of expansion of the voids (one continues expanding and one starts contracting) within each row and each column. An alternating pattern, this time based on void ovalization (with the orientation of the ovalized voids alternating this time) appears in the bifurcation mode in the compressive case. Needless to say, that once a finite wavelength bifurcation or a surface instability is encountered, the stability calculations for higher loads (which are based on the unit cell principal solution) are no longer meaningful due to the presence of bifurcated equilibrium branches in that load neighborhood.

The microgeometry-based comparison of the macroscopic onset-of-failure curves in strain space for porous elastomers with different arrangements of circular voids and an initial porosity $f_{0}=\pi / 16 \approx 0.2$ is presented in Fig. 5. In Fig. 5a are plotted, in principal logarithmic strain space, the macroscopic onset-of-failure (loss of ellipticity of the homogenized moduli) curves for a porous elastomer with three different microgeometries: (i) initially perfect square (solid lines), (ii) initially perfect hexagonal (dashed lines) and (iii) initially random polydisperse (dashed-dotted lines) arrangements of circular voids. The macroscopic strains corresponding to percolation and to zero porosity are also recorded (dashed-dotted-starred lines). A blow-up of the biaxial compression region of the above 
a

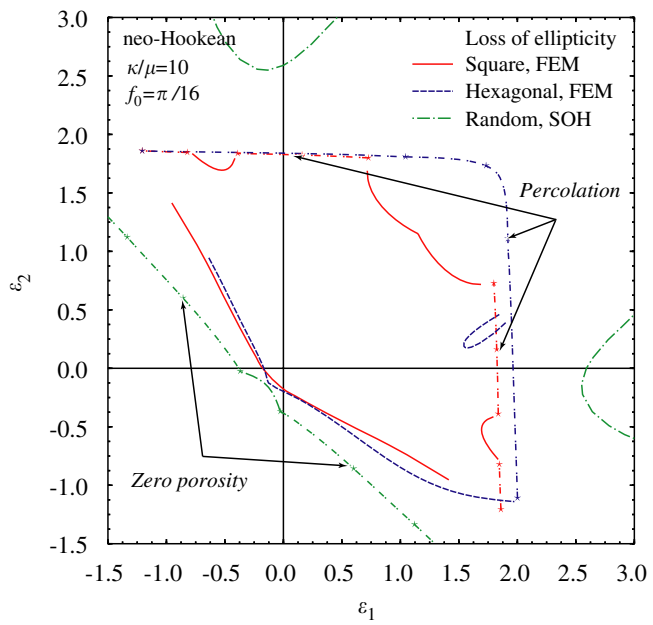

b

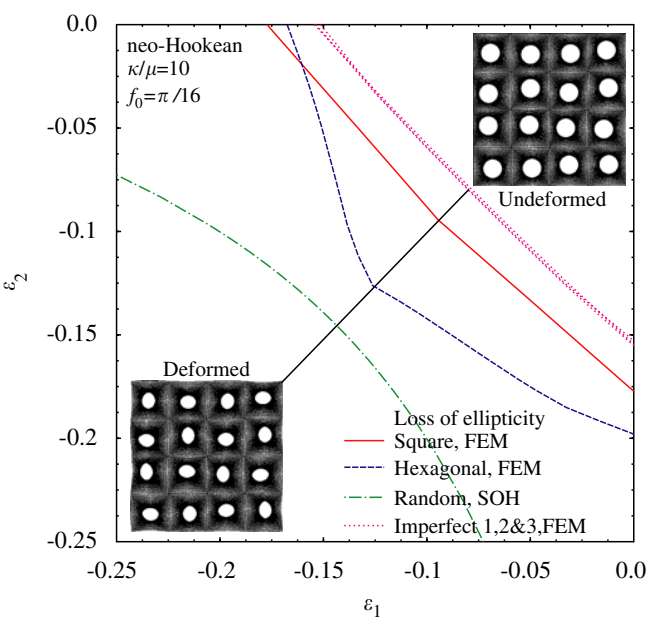

Fig. 5. In (a) is plotted, in principal logarithmic strain space, the microgeometry-based comparison of the macroscopic onset-of-failure curves in plane strain for a neo-Hookean matrix elastomer for three different microgeometries: (i) initially perfect square (solid lines), (ii) initially perfect hexagonal (dashed lines) and (iii) an initially random polydisperse (dashed-dotted lines) microgeometries. The macroscopic strains corresponding to percolation and to zero porosity are also recorded (dashed-dotted-starred lines). A blow-up of the biaxial compression region of the above graph is plotted in (b). Also in (b) are plotted the macroscopic onset-of-failure curves of three imperfect microgeometry elastomers using $4 \times 4$ "super cells", resulting by a geometric perturbation (with an imperfection amplitude $\xi=0.1$ ) of a $4 \times 4$ perfect square arrangement of unit cells, having one circular void each. The undeformed configuration of one such imperfect "super cell" and its deformed configuration at the onset of macroscopic failure plane strain equibiaxial compression $\left(\varepsilon_{1}=\varepsilon_{2} \leqslant 0\right)$ are also depicted.

graph is plotted in Fig. 5b. Results for the perfect and imperfect periodic microgeometries are based on FEM calculations, while the random microgeometry results are based on $\mathrm{SOH}$ calculations.

In comparing the macroscopic onset-of-failure curves for the square and hexagonal microgeometries, one notes that for load path angles in the approximate range $2 \pi / 3<\varphi<11 \pi / 6$ the square and hexagonal loss of ellipticity curves are not that far apart. For the remaining range, there are sizeable differences, the most noticeable being the absence of an instability for the hexagonal microstructure under biaxial tension. Note that the random microgeometry porous elastomer of the same porosity loses ellipticity under biaxial compression (for higher but comparable to its ordered counterpart macroscopic strains) and also in the neighborhood of uniaxial tension (for very large macroscopic strains, $\varepsilon_{\max }>2.5$ ). Percolation is found only for the ordered microgeometries, while pore closure is found for the random case for load path angles in the approximate range $3 \pi / 4<\varphi<\pi$ and $3 \pi / 2<\varphi<7 \pi / 4$. As expected, for a given load path the macroscopic failure for the ordered porous elastomers always occurs at a load parameter lower than the one corresponding to the random case with the same porosity and hence the macroscopic onset-of-failure curves for the random case are outside (and often at considerable distance) of their ordered microstructure counterparts. 
Also in Fig. 5b are plotted (dotted line) the macroscopic onset-of-failure curves of three imperfect microgeometry porous elastomers using $4 \times 4$ aggregates, resulting by a geometric perturbation, with an imperfection amplitude $\xi=0.1$, of a $4 \times 4$ perfect square arrangement of unit cells (the reference configuration of one such aggregate is shown in Fig. 5b). These macroscopic instabilities occur at strains almost identical to the ones shown in Fig. $4 \mathrm{~b}$ for the first microscopic instability of the infinite perfect porous elastomer with the square microstructure. The bifurcation load of the perfect $4 \times 4$ periodic structure (which coincides with the bifurcation load of the perfect $2 \times 2$ periodic structure) appears as a limit load for the corresponding imperfect $4 \times 4$ aggregate. Consequently, the microscopic instability curve in Fig. $4 \mathrm{~b}$ practically coincides with the loss of ellipticity of the imperfect $4 \times 4$ aggregate in Fig. 5b. Hence, a small perturbation of the periodic square microstructure results in a macroscopic failure at smaller strains while a large perturbation that results in a random microstructure with the same porosity results at a loss of ellipticity at much higher strains, as seen in Fig. 5b. An idea of the deformed configuration of the imperfect $4 \times 4$ aggregate at the onset of its macroscopic instability under equibiaxial compression is also shown in Fig. 5b, where one notices a deformation pattern close to the eigenmode of the corresponding perfect porous elastomer shown in Fig. 4b.

The microgeometry-based comparison of the macroscopic onset-of-failure curves in stress space, as well as the information about the corresponding loss of ellipticity directions

a

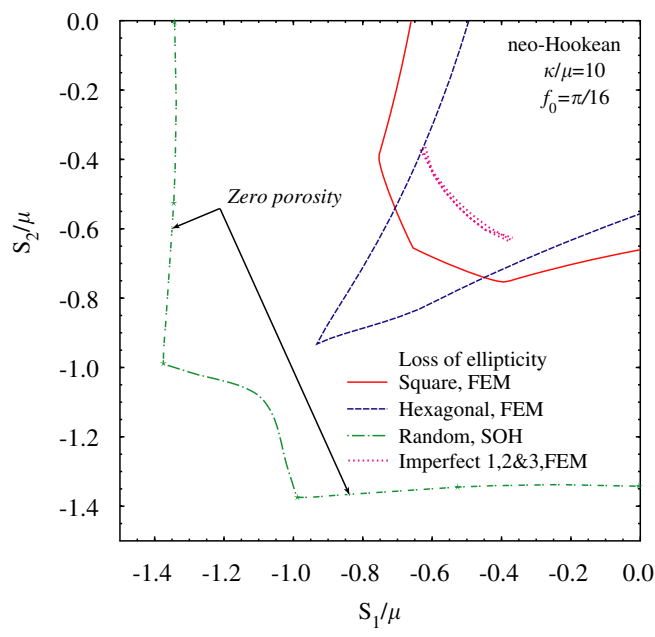

b

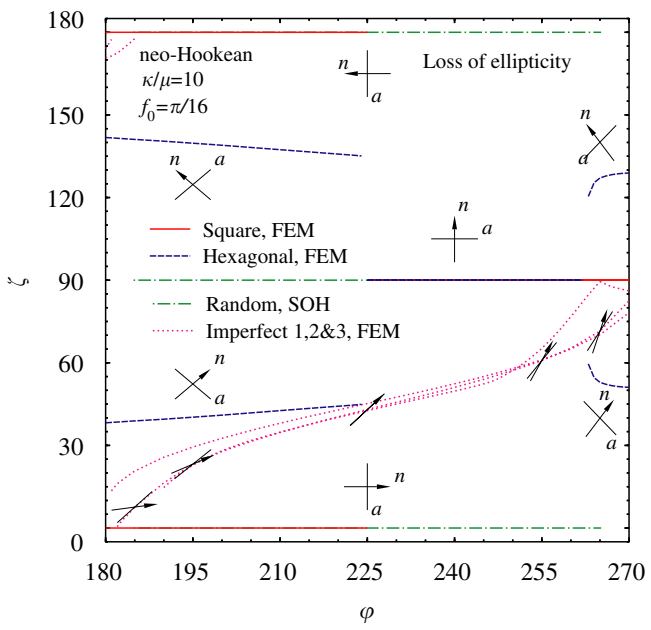

Fig. 6. In (a) is plotted, in dimensionless principal nominal stress space, the microgeometry-based comparison of the macroscopic instability curves for plane strain biaxial compression of a neo-Hookean matrix elastomer with (i) initially perfect (solid line) and (ii) imperfect with imperfection amplitude $\xi=0.1$ (dotted line) square, (iii) initially perfect hexagonal (dashed line) and (iv) initially random (dashed-dotted line) polydisperse microgeometries. In (b) are plotted, for the same elastomers, the angle $\zeta$ (angle between the $x_{1}$ axis and the normal $\mathbf{n}$ corresponding the homogenized moduli loss of ellipticity) as a function of the macroscopic load path angle $\varphi$. The relative orientation between the direction $\mathbf{n}$ and amplitude a (i.e., perpendicular or not) at the loss of ellipticity is also indicated. 
for the porous elastomers examined in Fig. 5 are presented in Fig. 6. In Fig. 6a are plotted, in dimensionless principal nominal stress space, the macroscopic instability (loss of ellipticity of the homogenized moduli) curves for biaxial compression of a solid with different microgeometries: (i) initially perfect (solid line) and (ii) imperfect with imperfection amplitude $\xi=0.1$ (dotted line) square, (iii) initially perfect hexagonal (dashed line) and (iv) initially random (dashed-dotted line) polydisperse arrangements of circular voids. As expected from the results in Fig. 5b and the stiffening of the porous elastomer under increasing straining in compression, the relative order of the curves is preserved in Fig. 6a. Hence, near equibiaxial compression, the hexagonal microstructure loses ellipticity at higher stresses than its square counterpart, while the trend reverses itself near uniaxial compression. Also the random microstructure always loses ellipticity at higher stresses than its ordered counterparts with the same porosity. The V-shape of the loss of ellipticity curve for the hexagonal microstructure is reminiscent of the corresponding results for aluminum honeycombs (see Triantafyllidis and Schraad, 1998). Notice that the macroscopic onset-of-failure curve for the imperfect porous elastomer with the square microstructure does not extend to the axes (the corresponding calculations are performed only for compressive strains in the range $\pi \leqslant \varphi \leqslant 3 \pi / 2$ ).

In Fig. $6 \mathrm{~b}$ are plotted, for the same porous elastomers and loading, the angle $\zeta$ (angle between the $X_{1}$ axis and the normal $\mathbf{n}$ (in the reference configuration) corresponding to the loss of ellipticity of the homogenized moduli) as a function of the macroscopic load path angle $\varphi$. The relative orientation between the direction $\mathbf{n}$ and amplitude a (i.e., perpendicular or not) at the loss of ellipticity is also indicated. For the random case, the loss of ellipticity always corresponds to the vanishing of the homogenized shear moduli $\left(\widetilde{L}_{1212}=\widetilde{L}_{2121}=0\right)$ and hence the loss of ellipticity orientation angle is $\zeta=k \pi / 2 ; k=0,1,2$. For the square microstructure, which shear modulus vanishes depends on the load path angle and hence $\zeta=0, \pi$ for $\pi \leqslant \varphi \leqslant 5 \pi / 4$ but $\zeta=\pi / 2$ for $5 \pi / 4 \leqslant \varphi \leqslant 3 \pi / 2$. For the hexagonal microstructure $\zeta=\pi / 2$ for a part of the $\varphi>5 \pi / 4$ range of the load path angle. As expected from the orthotropy of the microstructure and the loading, for the case of perfect periodic and random microstructures, the graphs are symmetric about the $\zeta=\pi / 2$ axis. For the imperfect square microstructure, the symmetry arguments are no longer valid and $\zeta$ is not only arbitrary, but also highly sensitive to the shape of the imperfection (recall that the three $4 \times 4$ aggregates have the same imperfection amplitude $\xi=0.1$ but different shapes).

The calculation method-based comparison of the macroscopic instability curves in strain space for porous elastomers with periodic (i) square and (ii) hexagonal, microgeometries and an initial porosity $f_{0}=\pi / 16 \approx 0.2$ are presented in Fig. 7 . In Fig. 7a and $\mathrm{b}$ are plotted for square and hexagonal microgeometry, respectively, in principal logarithmic strain space, the macroscopic onset-of-failure (loss of ellipticity of the homogenized moduli) curves periodic porous elastomers using two different calculation methods: FEM (dotted lines) and $\mathrm{SOH}$ (solid lines). The macroscopic strains corresponding to the onset of an instability at the free surface of the voids (dashed lines) are also recorded, as are the macroscopic strains corresponding to percolation and to zero porosity (dashed-dottedstarred lines).

Comparing the results of the two calculation methods in the biaxial tension quadrant $\varepsilon_{1}>0, \varepsilon_{2}>0$ is pointless in view of the large porosities involved and the resulting inadequacy of the $\mathrm{H}-\mathrm{S}$ approximation used in the $\mathrm{SOH}$ method, as previously discussed. For the remaining quadrants, the agreement of the two methods is remarkably better for 
a

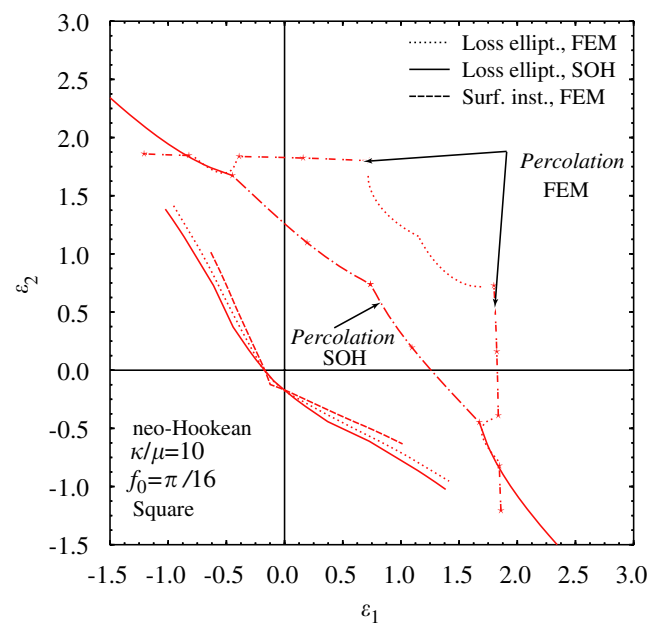

b

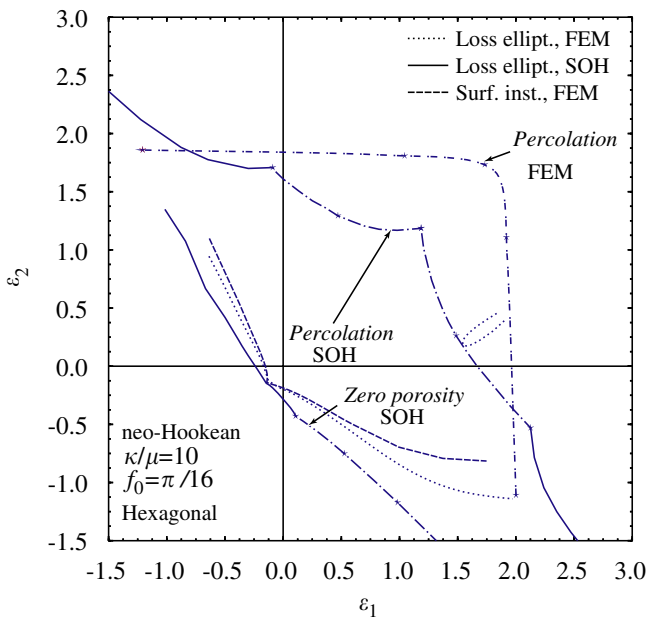

Fig. 7. In (a) is plotted, in principal logarithmic strain space, the calculation method-based comparison of the macroscopic onset-of-failure curves in plane strain for a neo-Hookean matrix elastomer for two different calculation methods: FEM (dotted lines) and $\mathrm{SOH}$ (solid lines) using an initially perfect square periodic microgeometry. The macroscopic strains corresponding to the onset of an instability at the free surface of the voids (dashed lines) are also recorded, as are the macroscopic strains corresponding to percolation and to zero porosity (dashed-dotted-starred lines). In (b) are plotted the corresponding results for the initially perfect hexagonal periodic microgeometry.

a

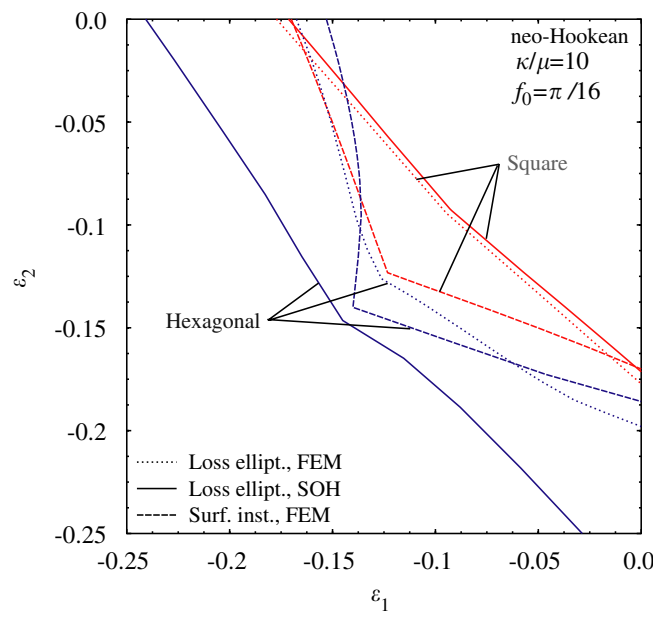

b

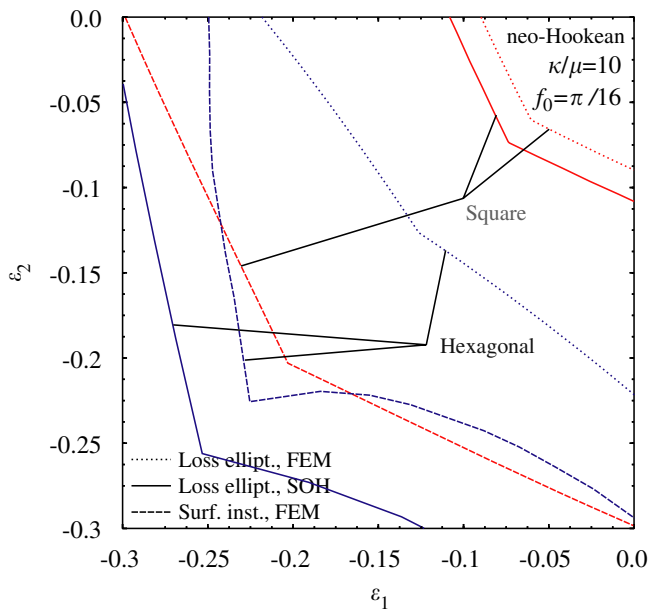

Fig. 8. Biaxial compression range blow-up of the results presented in Fig. 7 for porosity $f_{0}=\pi / 16(\approx 0.2)$ in (a) and corresponding results for porosity $f_{0}=0.5$ in (b).

the square microgeometry (see Fig. 7a). It is worth noticing that the SOH method gives a good approximation for the FEM loss of ellipticity even for the large strains in the mixed strains quadrants $\left(\varepsilon_{1} \varepsilon_{2}<0\right)$. The agreement of the two methods for the square 
microgeometry is remarkably good in the biaxial compression quadrant, as seen in the blow-up plot in Fig. 8a. For the considerably higher initial porosity $f_{0}=0.5$ and under biaxial compression, the $\mathrm{SOH}$ method gives a reasonable estimate for the FEM loss of ellipticity for the square microstructure, but an unsatisfactory overestimation for the hexagonal microstructure, as can be seen by comparing Fig. 8 b with a.

It should be pointed out that the FEM loss of ellipticity results are reliable as long as the unit cell calculations are accurate, thus requiring the knowledge of the macroscopic strains corresponding to the onset of a surface instability at the void. For all load path angles in the mixed strains quadrants, when a surface instability exists it precedes the macroscopic loss of ellipticity. In the biaxial compression range shown in Fig. 6, for the square microgeometry and almost all load path angles the loss of ellipticity occurs at lower strains than the surface instability, while for the hexagonal microgeometry, according to Fig. 6, this happens only for a small range of load paths near the equibiaxial compression. It is also worth noticing that the best agreement between the FEM and the $\mathrm{SOH}$ calculation methods occurs for the equibiaxial compression path, in view of the resulting isotropy of the solid as well as the small porosity and circular aspect ratio of the voids.

The remaining two figures of this subsection (Figs. 9,10) pertain to the influence of initial porosity on the macroscopic onset-of-failure curves of neo-Hookean solids. The first figure (Fig. 9) gives the results in macroscopic logarithmic strain space and the second (Fig. 10) in macroscopic nominal stress space. Only the biaxial compression regime is investigated.

The macroscopic onset-of-failure (loss of ellipticity of the homogenized moduli) curves in principal logarithmic strain space for biaxially compressed solids with three different

a

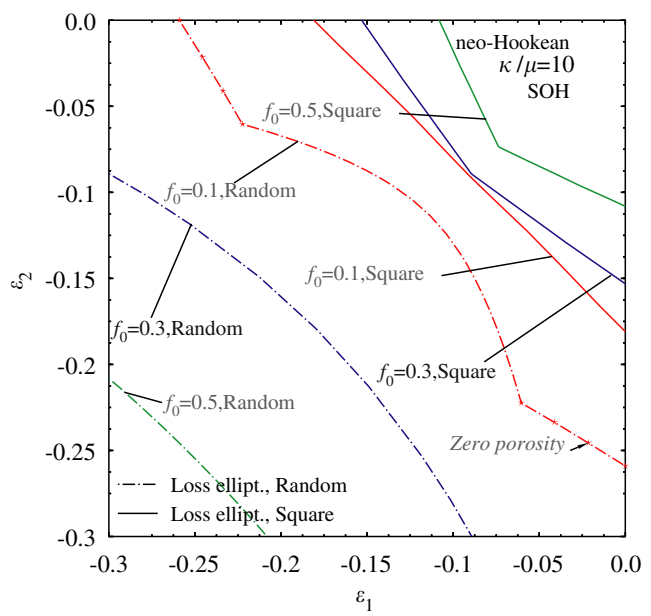

b

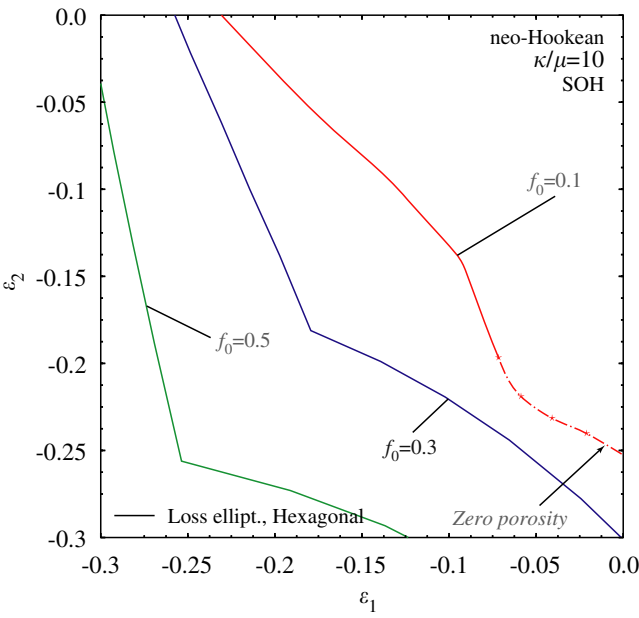

Fig. 9. Porosity influence on the macroscopic onset-of-failure curves in principal logarithmic strain space for plane strain biaxially compressed, neo-Hookean matrix elastomers with three different initial porosities $\left(0.1 \leqslant f_{0} \leqslant 0.5\right)$, using (i) initially perfect square and (ii) random polydisperse microgeometries in (a) and initially perfect hexagonal microgeometry in (b). 
a

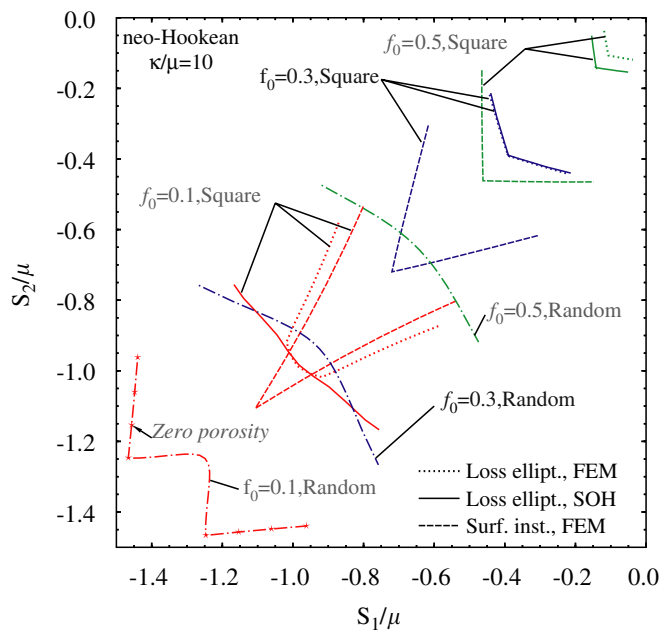

b

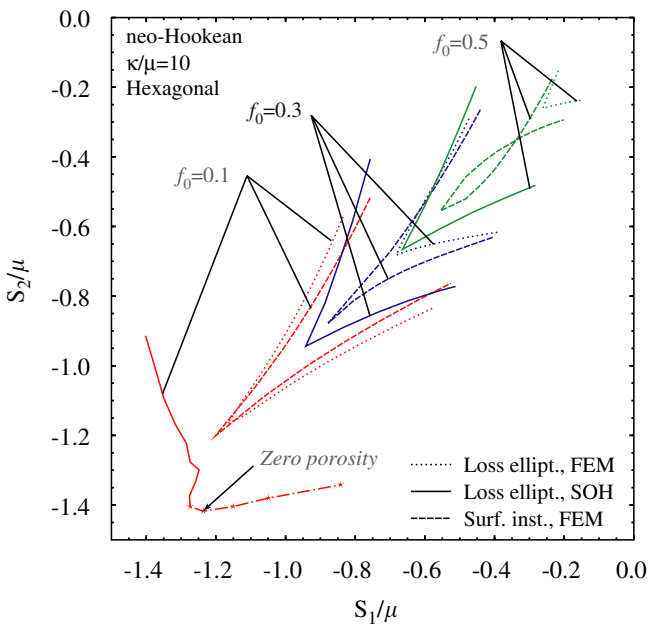

Fig. 10. Porosity influence on the macroscopic onset-of-failure curves in dimensionless principal nominal stress space, for plane strain biaxially compressed neo-Hookean matrix elastomers with three different initial porosities $\left(0.1 \leqslant f_{0} \leqslant 0.5\right)$, using (i) initially perfect square and (ii) random polydisperse microgeometries in (a) and initially perfect hexagonal microgeometry in (b).

initial porosities $\left(f_{0}=0.1,0.3,0.5\right)$, using (i) initially perfect square and (ii) random polydisperse arrangements of circular voids are plotted in Fig. 9a, while the corresponding results for initially perfect hexagonal arrangements of circular voids are plotted in Fig. 9b. For the periodic microgeometry the results are based on two different calculation methods: FEM (not shown due to graph overcrowding) and $\mathrm{SOH}$ (solid lines), while for the random microgeometry (dashed-dotted lines) the results are based on SOH. The macroscopic strains corresponding to the zero porosity curves (dashed-dotted-starred lines) are also recorded.

For the square microgeometry and for compressive loads, an increase in initial porosity results in thinner ligaments that are more prone to buckling, thus expecting a decrease in macroscopic critical strains. Indeed, Fig. 9a shows the SOH macroscopic instability curves nested one inside the other according to increasing initial porosity.

The rather counterintuitive results that the stability of the random microgeometry solid improves monotonically with increasing porosity can be explained from Fig. 10a in which the same results, plus the FEM loss of ellipticity and void surface instability curves, are plotted in stress space. The macroscopic onset-of-failure curves in stress space clearly show that, independently of the microgeometry and for a given load path, the critical stresses of a porous elastomer decrease monotonically with increasing porosity. As expected for the lower porosity $f_{0}=0.1$ and near equibiaxial compression there is an excellent agreement between the FEM and $\mathrm{SOH}$ results, while for the larger porosities $f_{0}=0.3,0.5$ the $\mathrm{SOH}$ overestimates the critical stresses.

In contrast to the square microgeometry case, the hexagonal SOH macroscopic onset-offailure curves in Fig. 9b, show an increase in stability with increasing porosity, exactly as for the random microgeometry in Fig. 9a. Moreover, for equibiaxial compression and for 
all porosities, there is a good agreement between the random and hexagonal SOH results, as expected from the hydrostatic stress state and the decreasing porosity of the essentially circular voids.

Again, the counterintuitive result that the stability of the hexagonal microgeometry solid improves monotonically with increasing porosity (according to the $\mathrm{SOH}$ results or even the FEM results for the two lower porosities $f_{0}=0.1,0.3$ ) can be explained from Fig. 10b in which the same results are plotted in stress space. The macroscopic onset-of-failure curves in principal nominal stress space clearly show that, independently of the microgeometry and for a given load path, the critical stresses of a porous elastomer decrease monotonically with increasing porosity. Once more, for the lower porosity $f_{0}=0.1$ there is a good agreement between the FEM and $\mathrm{SOH}$ results, while for the larger porosities $f_{0}=0.3,0.5$ the SOH substantially overestimates the critical strains. Note also that, only for the $f_{0}=0.5$ porous elastomer in biaxial compression, the surface instability always occurs at macroscopic stresses higher than the ones corresponding to the onset of the FEM macroscopic failure.

\subsection{Onset-of-failure curves for different matrix materials and load axes orientations}

The influence of the matrix constitutive law on the onset-of-failure curves in porous elastomers subjected to biaxial loading aligned with the axes of orthotropy is presented in Fig. 11, while the influence of the load axes orientation on the macroscopic onset-of-failure curves is presented in Fig. 12. All results in this subsection are calculated using an initial porosity of $f_{0}=\pi / 16 \approx 0.2$.

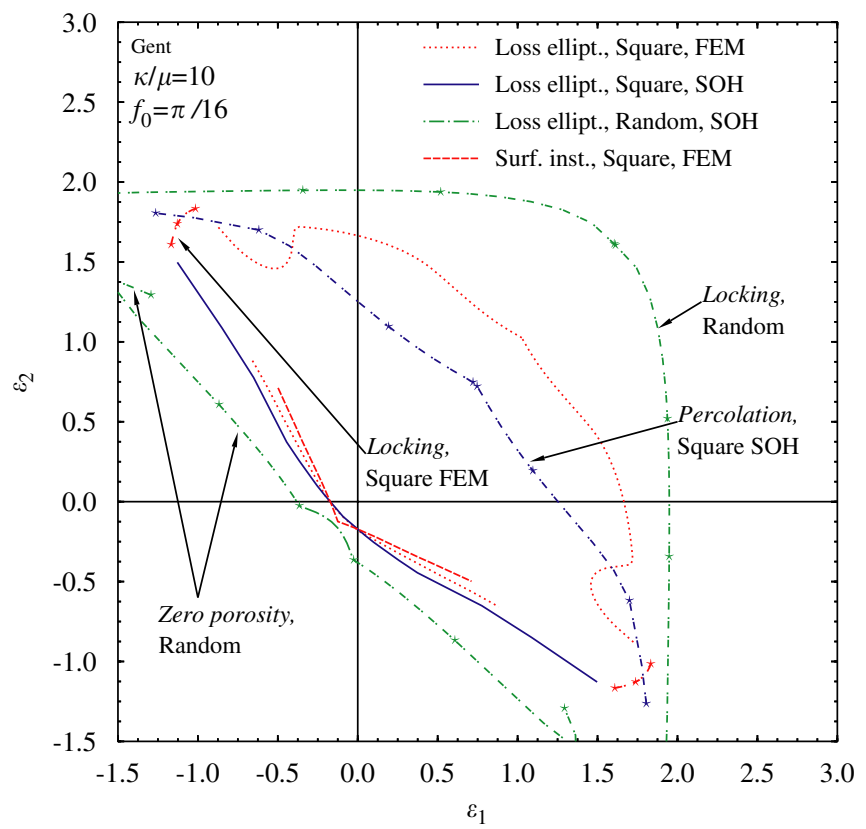

Fig. 11. Macroscopic onset-of-failure curves in principal logarithmic strain space, for a Gent matrix elastomer with (i) square and (ii) random polydisperse microgeometries. 


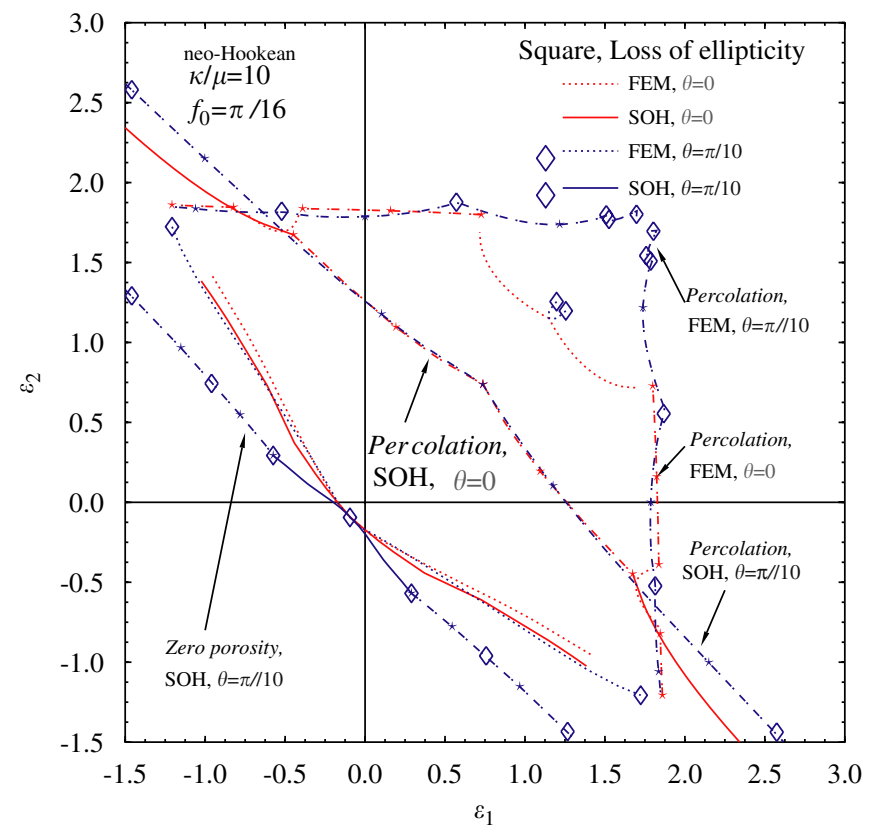

Fig. 12. Loading orientation influence on the macroscopic onset-of-failure curves, in principal logarithmic strain space, for neo-Hookean elastomer with an square microgeometry for macroscopic loadings that are (i) aligned $(\theta=0)$ and (ii) at an angle $(\theta=\pi / 10)$ with respect to initial axes of orthotropy.

In Fig. 11 are plotted, in principal logarithmic strain space, the macroscopic (loss of ellipticity of the homogenized moduli) onset-of-failure curves for a solid with a Gent matrix material $\left(\kappa / \mu=10, J_{m}=50\right)$, for (i) square and (ii) random polydisperse arrangements of circular voids. For the square microgeometry the results are based on FEM calculations (dotted lines) and on $\mathrm{SOH}$ calculations (solid lines), while for the random case the results are based only on $\mathrm{SOH}$ calculations (dashed-dotted lines). The macroscopic strains corresponding to the onset of an instability at the free surface of the voids (dashed lines), to percolation (dashed-dotted-starred lines) and to locking (dotted-starred lines) are also recorded.

As seen in Fig. 11, for the random case, the ellipticity is lost only in the biaxial compression regime while in the other regimes the material either locks or reaches zero porosity. The FEM results indicate that a loss of ellipticity is reached for all strain paths, except for the ones in the neighborhood of $\varepsilon_{1}=-\varepsilon_{2}$ where a percolation appears. The $\mathrm{SOH}$ results are close to their FEM counterparts for load path angles in the approximate range $3 \pi / 4<\varphi<7 \pi / 4$. For the same load paths a surface instability also exists but appears after the loss of ellipticity only in the biaxial compression regime.

A direct comparison of Fig. 7a and Fig. 11 shows no appreciable difference between the loss of ellipticity curves for the neo-Hookean and Gent solids because their response in the biaxial compression range is essentially the same.

All the results up to this point are calculated for loading with principal strains aligned with the axes of orthotropy $(\theta=0)$. In contrast, Fig. 12 addresses the loading orientation influence on the macroscopic instability of a neo-Hookean solid with $(\kappa / \mu=10)$, an initial 
porosity $f_{0}=\pi / 16 \approx 0.2$ and an initially perfect square arrangement of circular voids. More specifically, Fig. 12 compares, in principal logarithmic strain space, the onset-offailure curves for macroscopic loadings with principal strain axes aligned $(\theta=0)$ and at an angle $(\theta=\pi / 10)$ with respect to initial axes of orthotropy, with curves for the latter case marked by a $\diamond$. The results are based on two different calculation methods: FEM (dotted lines) and $\mathrm{SOH}$ (solid lines). The macroscopic strains corresponding to zero porosity and percolation are also plotted (dashed-dotted-starred lines).

Notice that outside the biaxial compression range, a slight change of principal strain axes orientation has a significant effect on the stability curves. Thus for biaxial tension, the macroscopic loss of ellipticity curve for the off-axes loading $(\theta=\pi / 10)$ almost disappears, except for a small segment near the equibiaxial path where the loading is essentially hydrostatic (and hence independent of the load axes orientation). What is also worth noticing is that the change of loading orientation has a considerable effect in the accuracy of the SOH approximation. Thus, for load paths in the approximate range $2 \pi / 3<\varphi<\pi$ and $3 \pi / 2<\varphi<11 \pi / 6$, while there is a satisfactory agreement between the FEM and SOH results for $\theta=0$, the $\mathrm{SOH}$ method considerably overpredicts the macroscopic onset-of-failure strains in spite of the fact that there is little difference of the FEM curves for $\theta=0$ and $\pi / 10$. Moreover, the $\mathrm{SOH}$ calculations for $\theta=\pi / 10$ predict pore closure prior to any macroscopic instability for loading paths at which the FEM results indicate a loss of ellipticity at considerably lower strains. In spite of the expected inaccuracy, the SOH method does predict the overall trends due to the change in the principal axes orientation of the macroscopic strain.

The above discussion gives a comprehensive picture of the influence of calculation method, microgeometry (porosity and void arrangement), matrix constitutive law and macroscopic load orientation on the microscopic and macroscopic onset-of-failure in hyperelastic porous elastomers under plane strain. A synthesis of these results is the object of the discussion presented in the next section.

\section{Discussion and conclusions}

The above-presented work is an in-depth study of the connections between microstructural instabilities and their macroscopic manifestations - as captured through the effective properties - in finitely strained porous elastomers. The powerful second-order homogenization $(\mathrm{SOH})$ technique, initially used for random media, is for the first time used here to study the onset of failure in periodic porous elastomers and the results are compared to more accurate finite element method (FEM) calculations. The influence of different microgeometries (random and periodic), initial porosity, matrix constitutive law and macroscopic load orientation on the microbuckling and macroscopic loss of ellipticity is investigated in detail. In addition to the above-described stability-based onset of failure mechanisms, limitations on the principal solution are also addressed, thus giving a complete picture of the different possible failure mechanisms present in finitely strained porous elastomers.

The most important stability feature of porous elastomers with a rank-one convex matrix and initially circular section pores under plane strain, is the consistent loss (at adequately large strains) of the rank-one convexity of the homogenized energy, independently of the microgeometric pore arrangement (random or periodic). This result should be contrasted with the case of elastomers that are reinforced with perfectly bonded circular section fibers. In this latter case, periodic fiber arrangements lead to macroscopic loss of ellipticity (Triantafyllidis et al., 2006), while random 
arrangements always give strongly elliptic responses (Lopez-Pamies and Ponte Castañeda, 2006b).

As expected from the general theory in Geymonat et al. (1993), microscopic bifurcation precedes the macroscopic loss of ellipticity for periodic microstructures. This occurs not only in biaxial compression but for square microgeometries in tension as well. For a fixed porosity, there are considerable differences between the macroscopic loss of ellipticity predictions of random and periodic media, especially when the deformation has one tensile principal strain. For biaxial compression, the failure curves are closer but always the failure along a given path happens at larger load parameters in the random medium than it is in periodic counterpart of the same porosity. There are also dramatic changes between the onset-of-failure curves for different periodic microgeometries.

Focusing attention on the most unstable (i.e., with the lowest critical strain or stress) region of biaxial compression, the critical stresses at the loss of ellipticity decrease monotonically with increasing initial porosity but this is not so for the corresponding critical strains. Of course, the validity of these onset-of-failure curves depends on checking a number of other limitations, i.e., void surface instability, percolation, strain lock-up and pore closure/self-contact.

In addition to the physically relevant results of the influence of different parameters on the failure of porous elastomers, the present investigation establishes the usefulness of the $\mathrm{SOH}$ techniques. This computational tool is not only capable of accurately predicting the macroscopic stress-strain response of finitely strained elastomers, but is powerful enough (because it allows for microstructure evolution) to predict loss of ellipticity in the effective medium, sometimes with great accuracy, as comparisons with FEM solutions show. In addition to its proved usefulness for the random case (where it is the only choice), the proposed methodology of using $\mathrm{SOH}$ techniques as a first approximation, showed itself to be extremely promising - and computationally more efficient than the FEM - for the study of the macroscopic response, microstructure evolution and stability of periodic elastomers as well. However, the FEM is the only method at this point that can be effectively used for the calculation of the microscopic instabilities of periodic elastomers (see Triantafyllidis et al., 2006). Nevertheless, the possibility of employing the $\mathrm{SOH}$ for the same purpose, although far from obvious at this stage, is worth investigating.

\section{Acknowledgments}

This research was carried out within the context of an NSF-CNRS international collaboration (NSF Grant No. OISE-0231867 and CNRS Grant No. 14555). The work of O.L.P. and P.P.C. was additionally supported by NSF Grant No. DMS-0204617.

\section{Appendix A. Constraints on the onset-of-failure curves}

As mentioned in Section 4, there are several other than loss of uniqueness or of rank-one convexity phenomena that can signal failure of the porous elastomer. They are (a) the instability that appears at large compressive strains on the free surface of the voids, (b) percolation, i.e., contact of adjacent pores leading to percolating network of voids, (c) strain lock-up, i.e., reaching of a maximum strain at the surface of the pores, which results in unrealistically stiff macroscopic response, and (d) pore self-contact (for the 
periodic case) or pore closure (for random microstructures), which dramatically alters the nature of the porous elastomer.

The surface instability of the voids is a relevant constraint only for periodic microstructures, since it signals - at finite macroscopic strains - the onset of local instabilities at the unit cell level, instabilities which are detected by the more accurate FEM-based calculations. For random microstructures, surface instability of the voids exists even for infinitesimally small macroscopic strains, due to strain concentration in the surface of the smallest pores. However, local instabilities are not accounted for in random microgeometry calculations. The remaining three limitations however, are applicable to any type of microgeometry.

A word of caution is needed when discussing constraints on the onset-of-failure curves related to the failure of the unit-cell solution for periodic microstructures. It pertains to the nucleation of voids at regions of high hydrostatic tension. Here only single-valued displacements are considered in the energy minimization problem discussed in Section 3 , thus excluding solutions which open holes in the matrix. The interested reader is referred to Ball (1982) for an in-depth discussion of such solutions. On the practical side, for the periodic porous elastomers considered here, hydrostatic tensions that are adequately high for void nucleation are not expected.

\section{A.1. Void surface instability}

The calculation of the microscopic onset-of-failure curves for periodic porous elastomers solid cannot be done analytically and relies here on the FEM technique. As previously explained, structural instability modes of the periodic porous solid can always be captured by a fine discretization of the unit cell. However, in solids with free surfaces that involve materials subjected to large strains there is a particular type of instability, termed surface instability, whose wavelength is infinitesimally small compared to any other characteristic geometric dimension of the problem and which decays exponentially away from the free surface. Fortunately this instability, first discussed by Biot (1965), is local in nature and can be detected analytically by checking the stretch ratio at each point of a free surface. The method works as follows.

Since the surface instability is a local phenomenon, it can be analyzed as the stability problem of a traction-free half-space subjected to a surface stretch ratio $\lambda_{\mathrm{s}}$. Without loss of generality the half-space is taken to be $X_{2}<0$. Due to its isotropy, the half-space has a constant deformation gradient $\mathbf{F}=\operatorname{diag}\left(\lambda_{i}\right)$ (with $\lambda_{1}=\lambda_{\mathrm{s}}$ ), where the normal stretch ratio $\lambda_{2}$ is found in terms of its tangential counterpart from the zero normal stress requirement $\sigma_{2}=0$. Under these assumptions, from (2.27), the equations governing the eigenmode at the onset of a surface instability of the homogeneous - and hence uniformly deformed along the principal directions $X_{i}$ - solid are

$$
L_{i j k l}\left(\lambda_{\mathrm{s}}\right) v_{k, l j}=0, \quad\left(X_{2}<0\right) ; \quad L_{i 2 k l}\left(\lambda_{\mathrm{s}}\right) v_{k, l}=0 \quad\left(X_{2}=0\right) ; \quad X_{1} \in R .
$$

To obtain the two differential equations and two boundary conditions (A.1) from (2.27) one notices that at the onset of an instability $\beta=0$, the outward normal to the free surface is $N_{i}=\delta_{i 2}$ and that the tangent moduli components $L_{i j k l}\left(\lambda_{\mathrm{s}}\right)$ are constants independent of the coordinates $X_{i}$. For such a system of linear differential equations with constant 
coefficients, the solution can be expressed as

$$
v_{j}=\sum_{k=1}^{2} v_{j}^{(k)}, \quad v_{j}^{(k)}=A_{j}^{(k)} \exp \left[\mathrm{i} \omega\left(X_{1}+r_{(k)} X_{2}\right)\right], \quad A_{2}^{(k)}=-A_{1}^{(k)} \frac{L_{1111}+r_{(k)}^{2} L_{1212}}{r_{(k)}\left(L_{1122}+L_{1221}\right)},
$$

where $r_{(k)}$ are the two roots with negative imaginary part of the biquadratic polynomial in $r$ :

$$
\begin{aligned}
& a r_{(k)}^{4}+2 b r_{(k)}^{2}+c=0, \quad \Im r_{(k)}<0, \quad(k=1,2) \\
& a \equiv L_{1212} L_{2222}, \quad 2 b \equiv L_{1212} L_{2121}+L_{1111} L_{2222}-\left(L_{1122}+L_{1221}\right)^{2}, \\
& c \equiv L_{1111} L_{2121} .
\end{aligned}
$$

The selection of the roots with negative imaginary part is dictated by the requirement of an exponential decay of the mode $\mathbf{v}$ away from the free surface, while the existence of roots with non-zero imaginary parts is guaranteed by the rank-one convexity of the solid assumed in (2.7). The reason for the biquadratic nature of the $r$-polynomial appearing in (A.3) is the orthotropy resulting in the initially isotropic solid when stressed along the $X_{i}$ axis.

Upon introduction of the eigenmode (A.2) into the two boundary conditions (A.1) $)_{2}$ one obtains a two-equation homogeneous linear system for the two unknowns $A_{1}^{(k)}, k=1,2$. A non-zero solution of the system requires the $2 \times 2$ matrix of coefficients of this linear system be singular:

$$
\operatorname{det}\left[\begin{array}{cc}
\frac{r_{(1)}^{2} L_{1122} L_{1212}-L_{1111} L_{1221}}{r_{(1)}\left(L_{1122}+L_{1221}\right)} & \frac{r_{(2)}^{2} L_{1122} L_{1212}-L_{1111} L_{1221}}{r_{(2)}\left(L_{1122}+L_{1221}\right)} \\
\frac{L_{2211} L_{2121}-r_{(1)}^{2} L_{2222} L_{2112}}{\left(L_{2121}+r_{(1)}^{2} L_{2222}\right)} & \frac{L_{2211} L_{2121}-r_{(2)}^{2} L_{2222} L_{2112}}{\left(L_{2121}+r_{(2)}^{2} L_{2222}\right)}
\end{array}\right]=0 .
$$

The above equation is a function of the surface stretch ratio $\lambda_{\mathrm{s}}$. Of interest are the two closest-to-unity roots, $\lambda_{\mathrm{s}}^{-}$and $\lambda_{\mathrm{s}}^{+}$, of (A.4) that signal the onset of a surface instability in compression and tension, respectively.

Thus, in addition to the microscopic and macroscopic onset-of-failure curves for periodic solids, an onset-of-surface-instability curve in macroscopic strain space can also be calculated. At each point of this curve there is at least one point in the hole where in the surface strain has reached one of the critical values $\lambda_{\mathrm{s}}^{-}$or $\lambda_{\mathrm{s}}^{+}$. For macroscopic strains inside this curve, no instability at the surface of the void is possible in the periodic porous elastomer in question.

\section{A.2. Percolation}

The evolution along finite strain loading paths of the size, shape, and orientation of the pores in the elastomers considered here may result into adjacent pores coming into contact, leading to a percolating network of voids. A different modeling approach of this 
phenomenon is required for calculations based on the $\mathrm{SOH}$ and on the FEM methods, as explained below.

\section{A.2.1. Percolation thresholds for second-order variational estimates}

As mentioned in Section 3.2.1, the H-S estimates for the LCC given by (3.12) are known to be accurate for small to moderate porosities, but they become increasingly inaccurate, and ultimately meaningless, for porosity levels near the percolation limit, when the interactions among the vacuous inclusions become especially strong. In addition, the underlying microstructure in the porous elastomers studied here evolves as a function of the applied finite deformation $\overline{\mathbf{F}}(\lambda)$. Consequently, the second-order estimates of the $\mathrm{H}-\mathrm{S}$ type should not be used once the relevant microstructural variables (which evolve along the loading path of choice) reach values approaching the percolation limit.

For the random microgeometry, following Ponte Castañeda and Willis (1995), the H-Stype second-order estimates utilized in this work are taken to become unsound whenever the pores penetrate the security distributional ellipses surrounding them. More precisely, the $\mathrm{H}-\mathrm{S}$-type second-order estimates for porous elastomers with the random isotropic microgeometry first become invalid whenever the deformed elliptical void comes into contact with the surrounding ellipse serving to characterize the distribution of the pores in the deformed configuration. Consistent with this definition of the domain of validity, it is important to emphasize that the $\mathrm{H}-\mathrm{S}$-type second-order estimates for the random microgeometry may become inappropriate before percolation occurs in a strict sense, even for aligned loading paths.

For the periodic square and hexagonal microgeometries considered here, the H-S-type second-order estimates become unsound whenever the pores penetrate the periodic cells surrounding them. For instance, the H-S-type second-order estimates for porous elastomers with the periodic square microgeometry first become invalid whenever the deformed elliptical void comes into contact with the surrounding parallelogram serving to characterize the distribution of the pores in the deformed configuration. In line with the above definition of rigorous validity, it is important to note that the second-order estimates of the H-S-type for the periodic (square and hexagonal) microgeometries become invalid, in general, before percolation actually takes place. However, under loading paths aligned with the axes of orthotropy (i.e., for $\theta=0$ and $\pi / 2$ ), it is not difficult to check that the points at which the second-order estimates first become invalid coincide with percolation.

Explicit percolation conditions, in terms of the macroscopic principal stretch ratios $\left(\lambda_{i}\right)$, the current porosity $(f)$ and current aspect ratio $(a)$, for the $\mathrm{H}-\mathrm{S}$-type second-order estimates defined by (3.3) and (3.12), are given for all three porous elastomer microstructures considered in this work. These conditions assume a loading path aligned with the axes of orthotropy (i.e., for $\theta=0$ and $\pi / 2$ ).

(i) Random isotropic distribution: The $\mathrm{H}-\mathrm{S}$ second-order estimates for porous elastomers with the random isotropic microgeometry subjected to aligned loading conditions first become invalid whenever one of the following equalities holds:

$$
\text { (i) } f=\frac{\lambda_{1}}{\lambda_{2}} a \text { or } \quad \text { (ii) } f=\frac{\lambda_{2}}{a \lambda_{1}} \text {. }
$$

Unlike the expressions for the periodic square (A.6) and hexagonal (A.7) distributions, conditions (A.5) do not necessarily correspond to percolation. However, in the undeformed configuration, it is easy to see that conditions (A.5) reduce to $f=1$, which 
happens to correspond exactly with the actual onset of percolation, within the context of this type of approximation.

(ii) Periodic square distribution: The $\mathrm{H}-\mathrm{S}$ second-order estimates for porous elastomers with the periodic square microgeometry subjected to a loading path aligned with the axes of orthotropy first become invalid whenever one of the following equalities holds:

$$
\text { (i) } f=\frac{\pi}{4} \frac{\lambda_{1}}{\lambda_{2}} a \text { or } \quad \text { (ii) } f=\frac{\pi}{4 a} \frac{\lambda_{2}}{\lambda_{1}} \text {. }
$$

For clarity, it is recalled here that the pore aspect ratio $a=z_{2} / z_{1}$, where $1 / z_{i}(i=1,2)$ denote the square roots of the eigenvalues of $Z_{k i} Z_{k j}$, (where $\mathbf{Z}=\left(\overline{\mathbf{F}}^{(2)}\right)^{-1}$ is the inverse of the average deformation gradient of the pore). Conditions (A.6) correspond to percolation in the context of the approximations associated with this type of estimate. In this connection, it is interesting to note that in the undeformed configuration (i.e., for $\lambda_{1}=\lambda_{2}=1$ ) $a=1$ and percolation occurs at a porosity of $f=f_{0}=\pi / 4$ (which agrees with the exact result).

(iii) Periodic hexagonal distribution: The $\mathrm{H}-\mathrm{S}$ second-order estimates for porous elastomers with the periodic hexagonal microgeometry subjected to a loading path aligned with the axes of orthotropy, first become invalid whenever one of the following equalities holds:

$$
\text { (i) } f=\frac{\pi}{2 \sqrt{3}} \frac{\lambda_{1}}{\lambda_{2}} a \quad \text { or } \quad \text { (ii) } f=\frac{\pi}{8 \sqrt{3}}\left(\frac{3}{a} \frac{\lambda_{2}}{\lambda_{1}}+\frac{\lambda_{1}}{\lambda_{2}} a\right) \text {, }
$$

where $a$ is again the pore aspect ratio. Conditions (A.7) correspond precisely to percolation, in the context of the approximations introduced in Section 3.2.2. Similar to the previous case, it is interesting to note that in the undeformed configuration percolation take place at a porosity of $f=f_{0}=\pi /(2 \sqrt{3})$ (which again agrees with the correct result).

\section{A.2.2. Percolation thresholds for FEM calculations}

For the periodic microgeometries where the deformed configuration is calculated using the FEM, a percolation is said to occur (in the principal solution) if the current thickness of the thinnest ligament between two adjacent voids becomes negligible compared the characteristic dimension of the deformed unit cell. More specifically, the FEM-based percolation criterion adopted here is

$$
\frac{d_{\min }}{l_{\max }} \leqslant t_{\text {perc }},
$$

where $d_{\min }$ and $l_{\max }$ are, respectively, the minimum ligament thickness between two adjacent voids and the maximum distance between two adjacent voids, as measured in the deformed principal solution. The numerical value for the percolation threshold $t_{\text {perc }}$ is taken in the following calculations to be $t_{\text {perc }}=0.005$. Although this choice is somewhat arbitrary, numerical experimentation with lower threshold values showed no appreciable difference in the percolation predictions in macroscopic strain space, thus explaining this choice.

\section{A.3. Strain lock-up}

A characteristic behavior of natural rubbers is their inability to sustain strains above a certain level, a phenomenon termed strain lock-up. When strains approach these limits, 
stresses rise sharply and consequently the values of the tangent moduli show dramatic increases. For porous elastomers with a matrix material that exhibits strain lock-up, the same dramatic increase in the values of the homogenized tangent moduli is expected as limiting values of strain are approached in the matrix. A numerical lock-up criterion for the SOH-homogenized elastomer is adopted based on the ratio between current $($ at $\overline{\mathbf{F}}$ ) and initial (at I) shear components of the effective moduli:

$$
\frac{\widetilde{\mathbf{L}}_{1212}(\overline{\mathbf{F}})}{\widetilde{\mathbf{L}}_{1212}(\mathbf{I})} \geqslant t_{\text {lock}},
$$

where $\widetilde{\mathbf{L}}$ is the effective moduli tensor for the porous elastomer defined in (2.10). For the case of periodic solids where the principal solution is obtained using the FEM, a slightly different criterion using their one-cell homogenized counterparts $\widehat{\mathbf{L}}=\mathbf{L}^{\mathrm{H}}$, with the latter calculated using (2.18) is employed:

$$
\max _{i, j, k, l} \frac{\widehat{\mathbf{L}}_{i j k l}(\overline{\mathbf{F}})}{\widehat{\mathbf{L}}_{i j k l}(\mathbf{I})} \geqslant t_{\text {lock }} .
$$

The numerical value for the lock-up threshold $t_{\text {lock }}$ is taken for all the following calculations to be $t_{\text {lock }}=10$. Once again, this choice is arbitrary, but numerical experimentation with lower threshold values showed no appreciable difference in the lock-up predictions in macroscopic strain space, thus supporting this choice.

\section{A.4. Pore closure/self-contact}

The last in the list of limitations associated with the solution of the finitely strained porous elastomer, pertains to the closure or to the self-contact of the void's free surface. When a porous elastomer is strained along a load path that decreases the elastomer's macroscopic volume, i.e., when the sum of the macroscopic principal logarithmic strains is negative $\left(\varepsilon_{1}+\varepsilon_{2}<0\right)$, its porosity decreases. For such load paths, the calculations based on the SOH method, for the homogenized (random microgeometry) or for the unit cell (periodic microgeometry) solution, eventually give a vanishing porosity $(f=0)$ at adequately large values of the load parameter $\lambda$. For periodic microgeometries calculated using the FEM the porosity never vanishes $(f>0)$, but at adequately large values of the load parameter $\lambda$ opposite faces of the void might come to contact, at which point the corresponding macroscopic strains are recorded and the calculations are terminated. Although pore self-contact has not been found for the neo-Hookean and Gent solids used in the FEM calculations presented here, pore self-contact occurs in periodic elastomers with softer matrix constitutive laws (unpublished work) and for this reason the FEM code used is capable of detecting this phenomenon.

\section{Appendix B. Expressions for the microstructural tensor $\mathbf{P}$}

In this appendix, we provide explicit expressions for the in-plane components of the tensor $\mathbf{P}$, which serve to characterize the three types of microstructures (in the reference configuration) considered in this work: periodic square, periodic hexagonal, and random isotropic distribution of aligned cylindrical pores with initially circular cross section. 


\section{B.1. Periodic square distribution}

The microstructural tensor $\mathbf{P}$ for the square distribution of cylindrical fibers with circular cross section may be written as (see Suquet, 1990a, b)

$$
P_{i j k l}=\frac{1}{\pi\left(1-f_{0}\right)} \sum_{\substack{p=-\infty \\-\{p=q=0\}}}^{+\infty} \sum_{\substack{q=-\infty \\-\infty}}^{+\infty}\left(L_{0 i m k n} \xi_{m} \xi_{n}\right)^{-1} \xi_{j} \xi_{l} \frac{J_{1}^{2}\left(2 \sqrt{\pi f_{0}} \sqrt{p^{2}+q^{2}}\right)}{p^{2}+q^{2}},
$$

where $\xi_{1}=p, \xi_{2}=q$, and $J_{1}(\cdot)$ is the Bessel function of first kind.

\section{B.2. Periodic hexagonal distribution}

The microstructural tensor $\mathbf{P}$ for the hexagonal distribution of cylindrical fibers with circular cross section may be written as (see Suquet, 1990a, b)

$$
P_{i j k l}=\frac{\sqrt{3}}{2 \pi\left(1-f_{0}\right)} \sum_{\substack{p=-\infty \\-\{p=q=0\}}}^{+\infty}\left(L_{0 i m k n} \xi_{m} \xi_{n}\right)^{-1} \xi_{j} \xi_{l} \frac{J_{1}^{2}\left(\frac{2^{3 / 2}}{3^{1 / 4}} \sqrt{\pi f_{0}} \sqrt{p^{2}-p q+q^{2}}\right)}{p^{2}-p q+q^{2}},
$$

where $\xi_{1}=p, \xi_{2}=\sqrt{3} / 3(2 q-p)$, and $J_{1}(\cdot)$ is the Bessel function of first kind.

\section{B.3. Random isotropic distribution}

The microstructural tensor $\mathbf{P}$ for the random isotropic distribution of cylindrical fibers with circular cross section may be expressed as (see Willis, 1977)

$$
P_{i j k l}=\frac{1}{2 \pi} \int_{0}^{2 \pi}\left(L_{0 i m k n} \xi_{m} \xi_{n}\right)^{-1} \xi_{j} \xi_{l} \mathrm{~d} \theta
$$

where $\xi_{1}=\cos \theta$ and $\xi_{2}=\sin \theta$.

\section{References}

Abeyaratne, R., Triantafyllidis, N., 1984. An investigation of localization in a porous elastic material using homogenization theory. J. Appl. Mech. 51, 481-486.

Ball, J.M., 1977. Convexity conditions and existence theorems in nonlinear elasticity. Arch. Ration. Mech. Anal. 63, 337-403.

Ball, J.M., 1982. Discontinuous equilibrium solutions and cavitation in nonlinear elasticity. Philos. Trans. R. Soc. London Ser. A 306, 557-611.

Biot, M.A., 1965. Mechanics of Incremental Deformation. Wiley, New York.

Blatz, P.J., Ko, W.L., 1962. Application of finite elastic theory to the deformation of rubbery materials. Trans. Soc. Rheol. 6, 223-251.

Braides, A., 1985. Homogenization of some almost periodic coercive functionals. Rend. Accad. Naz. XL 9, 313-322.

Geymonat, G., Müller, S., Triantafyllidis, N., 1993. Homogenization of nonlinearly elastic materials, microscopic bifurcation and macroscopic loss of rank-one convexity. Arch. Ration. Mech. Anal. 122, 231-290.

Gong, L., Kyriakides, S., Triantafyllidis, N., 2005. On the stability of kelvin cell foams under compressive loads. J. Mech. Phys. Solids 53, 771-794.

Hill, R., 1972. On constitutive macro-variables for heterogeneous solids at finite strain. Proc. Roy. Soc. A 326 , $131-147$. 
Knowles, J.K., Sternberg, E., 1975. On the ellipticity of the equations of nonlinear elastostatics for a special material. J. Elasticity 5, 341-361.

Laws, N., 1973. On the thermostatics of composite materials. J. Mech. Phys. Solids 21, 9-17.

Lopez-Pamies, O., Ponte Castañeda, P., 2003. Second-order estimates for the macroscopic for the large deformation response of particle reinforced rubbers. C.R. Mec. 331, 1-8.

Lopez-Pamies, O., Ponte Castañeda, P., 2004a. Second-order homogenization estimates incorporating field fluctuations in finite elasticity. Math. Mech. Solids 9, 243-270.

Lopez-Pamies, O., Ponte Castañeda, P., 2004b. Second-order estimates for the macroscopic response and loss of ellipticity in porous rubbers at large deformations. J. Elasticity 76, 247-287.

Lopez-Pamies, O., Ponte Castañeda, P., 2006a. On the overall behavior, microstructure evolution, and macroscopic stability in reinforced rubbers at large deformations: I-Theory. J. Mech. Phys. Solids 54, 807-830.

Lopez-Pamies, O., Ponte Castañeda, P., 2006b. On the overall behavior microstructure evolution, and macroscopic stability in reinforced rubbers at large deformations: II-Application to cylindrical fibers. J. Mech. Phys. Solids 54, 831-863.

Malvern, L.E., 1969. Introduction to the Mechanics of a Continuous Medium. Prentice-Hall, Englewood Cliffs, NJ.

Marcellini, P., 1978. Periodic solutions and homogenization of nonlinear variational problems. Ann. Mat. Pura Appl. 4, 139-152.

Müller, S., 1987. Homogenization of nonconvex integral functionals and cellular elastic materials. Arch. Ration. Mech. Anal. 99, 189-212.

Nemat-Nasser, S., Iwakuma, T., Hejazi, M., 1982. On composites with periodic structure. Mech. Mater. 1, 239-267.

Nestorović, M., Triantafyllidis, N., 2004. Onset of failure in finitely strained layered composites subjected to combined normal and shear loading. J. Mech. Phys. Solids 52, 941-974.

Ponte Castañeda, P., 1989. The overall constitutive behavior of nonlinear elastic composites. Proc. R. Soc. A 422, $147-171$.

Ponte Castañeda, P., 1996. Exact second order estimates for the effective mechanical properties of nonlinear composite materials. J. Mech. Phys. Solids 44, 827-862.

Ponte Castañeda, P., 2002. Second-order homogenization estimates for nonlinear composites incorporating field fluctuations. I. Theory. J. Mech. Phys. Solids 50, 737-757.

Ponte Castañeda, P., Willis, J.R., 1995. The effect of spatial distribution on the effective behavior of composite materials and cracked media. J. Mech. Phys. Solids 43, 1919-1951.

Schraad, M.W., Triantafyllidis, N., 1997. Scale effects in media with periodic and nearly periodic microstructures, II-Failure mechanisms. J. Appl. Mechanics 64, 763-771.

Suquet, P., 1990a. Une méthode simplifiée pour le calcul des propriétés élastiques de matériaux hétérogènes à structure périodique. C. R. Acad. Sci. Paris 311, 769-774.

Suquet, P., 1990b. Méthodes de calcul simplifiées pour la détermination des propriétés élastiques linéaires de composites à structure périodique. Internal Report, L.M.A. Marseille.

Triantafyllidis, N., Bardenhagen, S.G., 1996. The influence of scale size on the stability of periodic solids and the role of associated higher order gradient continuum models. J. Mech. Phys. Solids 44, 1891-1928.

Triantafyllidis, N., Maker, B.N., 1985. On the comparison between microscopic and macroscopic instability mechanisms in a class of fiber-reinforced composites. J. Appl. Mech. 52, 794-800.

Triantafyllidis, N., Schraad, M.W., 1998. Onset of failure in aluminum honeycombs under general in-plane loading. J. Mech. Phys. Solids 46, 1089-1124.

Triantafyllidis, N., Nestorović, M.D., Schraad, M.W., 2006. Failure surfaces for finitely strained two-phase periodic solids under general in-plane loading. J. Appl. Mech. 73, 505-515.

Willis, J.R., 1977. Bounds and self-consistent estimates for the overall moduli of anisotropic composites. J. Mech. Phys. Solids 25, 185-202. 